\title{
Divergent Synthesis of Complex Polyketide-Like Macrolides from a Simple Polyol Fragment
}

\author{
Michael J. Zacuto and James L. Leighton* \\ Department of Chemistry, Columbia University, New York, New York, 10027 \\ Supporting Information
}

General Information. Degassed solvents were purified by passage through an activated alumina column. Diisopopylamine and triethylamine were distilled from $\mathrm{CaH}_{2}$ prior to use. All other commercially obtained reagents were used as received. Infrared spectra were recorded on a Perkin Elmer Paragon 1000 FT-IR spectrometer. Low-resolution mass spectra were obtained on JEOL HX110 mass spectrometer in the Columbia University Mass Spectrometry Laboratory. Optical rotations were recorded on a Jasco DIP-1000 digital polarimeter. ${ }^{1} \mathrm{H}$ NMR spectra were recorded on a Bruker DPX-500 (500 MHz), a Bruker DPX-400 (400 MHz) and a Bruker DPX-300 (300 MHz) spectrometers and are reported in ppm from $\mathrm{CDCl}_{3}$ internal standard (7.26 ppm). Data are reported as follows: $(\mathrm{s}=$ singlet, $\mathrm{br} \mathrm{s}=$ broad singlet, $\mathrm{d}=$ doublet, $\mathrm{t}=$ triplet, $\mathrm{q}=$ quartet, quin $=$ quintet, $\mathrm{sep}=$ septet, $\mathrm{m}=$ multiplet, $\mathrm{dd}=$ doublet of doublets, $\mathrm{td}=$ triplet of doublets, $\mathrm{tt}=$ triplet of triplets, $\mathrm{dq}=$ doublet of quartets, ddt $=$ doublet of doublet of triplets; coupling constant(s) in Hz; integration). Proton decoupled ${ }^{13} \mathrm{C}$ NMR spectra were recorded on Bruker DPX-500 (125 MHz), a Bruker DPX-400 (100 MHz) and a Bruker DPX-300 (75 MHz) spectrometers and are reported in ppm from $\mathrm{CDCl}_{3}$ internal standard $(77.0 \mathrm{ppm})$.

\section{Experimental Procedures:}

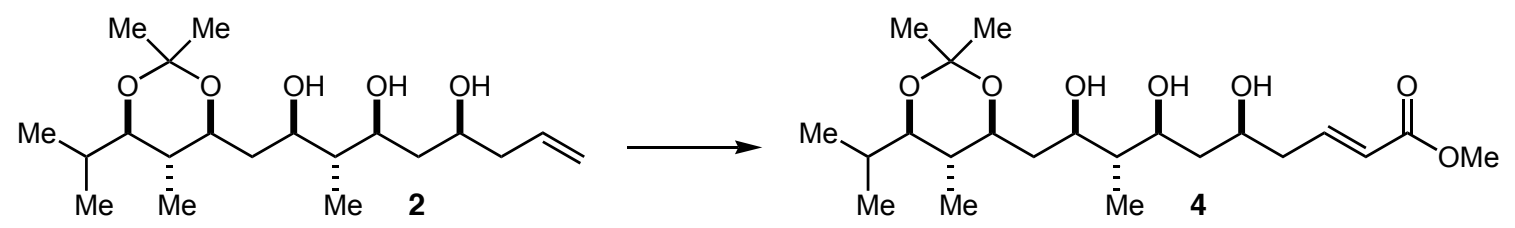

Ester 4. Tricylcohexylphosphine[1,3-bis(2,4,6-trimethylphenyl)-4,5-dihydroimidazol-2-ylidene][benzylidene] ruthenium (IV) dichloride $(6 \mathrm{mg}, 0.008 \mathrm{mmol})$ was weighed out in a glove box, then taken outside the box and dissolved in $1.0 \mathrm{~mL}$ of $\mathrm{CH}_{2} \mathrm{Cl}_{2}$. The resulting red solution was added by canula to a solution of 2 (58 $\mathrm{mg}, 0.162 \mathrm{mmol})$ and methyl acrylate $(0.4 \mathrm{~mL}, 4.4 \mathrm{mmol})$ in $1.0 \mathrm{~mL}$ of $\mathrm{CH}_{2} \mathrm{Cl}_{2}$. A reflux condenser was 
attached to the reaction flask and heating to reflux began. After 14 hours, TLC analysis indicated complete consumption of 2 . Following concentration, the residue was subjected to silica gel chromatography, initially eluting with a slow gradient of $0 \rightarrow 20 \%$ EtOAc/Hexanes in order to separate the major impurities (mainly methyl acrylate derivatives). Increasing the EtOAc concentration to $25 \rightarrow 30 \%$ allowed for the isolation of 4 (62 $\mathrm{mg}, 93 \%$ yield $)$ as a clear colorless oil. ${ }^{1} \mathrm{H} \mathrm{NMR}\left(500 \mathrm{MHz}, \mathrm{CDCl}_{3}\right) \delta$ 7.06-6.99 (m, 1H, $\left.\mathrm{CH}=\mathrm{CHCO}_{2} \mathrm{CH}_{3}\right), 5.90$ $\left(\mathrm{d}, J=15.7 \mathrm{~Hz}, 1 \mathrm{H}, \mathrm{CH}=\mathrm{CHCO}_{2} \mathrm{CH}_{3}\right), 4.04-3.98(\mathrm{~m}, 1 \mathrm{H}, \mathrm{CHOR}), 3.95-3.87$ (m, 1H, CHOR), 3.75-3.68 (m, 5H, two CHOR and $\left.\mathrm{OCH}_{3}\right), 3.35\left(\mathrm{dd}, J_{1}=10.3 \mathrm{~Hz}\right.$ and $\left.J_{2}=1.8 \mathrm{~Hz}, 1 \mathrm{H},\left(\mathrm{CH}_{3}\right)_{2} \mathrm{CHCHOR}\right), 2.45-2.34(\mathrm{~m}, 2 \mathrm{H}$, $\left.\mathrm{CH}_{2} \mathrm{CH}=\mathrm{CHCO}_{2} \mathrm{CH}_{3}\right), 2.05-1.99\left(\mathrm{~m}, 1 \mathrm{H}, \mathrm{CH}_{2}\right), 1.89-1.83\left(\mathrm{~m}, 1 \mathrm{H}, \mathrm{CH}\left(\mathrm{CH}_{3}\right)_{2}\right), 1.70-1.63(\mathrm{~m}, 1 \mathrm{H}, \mathrm{CH}), 1.55-$ 1.39 (m, 7H, two $\mathrm{CH}_{2}$ and two $\mathrm{CHCH}_{3}$ and one $\left.\mathrm{ROC}\left(\mathrm{CH}_{3}\right)_{2} \mathrm{OR}\right), 1.35$ (s, 3H, one $\left.\mathrm{ROC}(\mathrm{CH})_{2} \mathrm{OR}\right), 0.93(\mathrm{~d}, J=$ $\left.6.9 \mathrm{~Hz}, 3 \mathrm{H}, \mathrm{CH}_{3}\right), 0.83\left(\mathrm{~d}, J=6.8 \mathrm{~Hz}, 3 \mathrm{H}, \mathrm{CH}_{3}\right), 0.77\left(\mathrm{~d}, J=7.0 \mathrm{~Hz}, 3 \mathrm{H}, \mathrm{CH}_{3}\right), 0.75\left(\mathrm{~d}, J=6.7 \mathrm{~Hz}, 3 \mathrm{H}, \mathrm{CH}_{3}\right)$; ${ }^{13} \mathrm{C}$ NMR $\left(100 \mathrm{MHz}, \mathrm{CDCl}_{3}\right) \delta 166.9,145.9,123.0,98.1,78.4,77.5,77.2,77.0,71.1,51.4,44.8,40.5,39.9$, $37.9,36.2,30.0,28.0,19.9,19.7,14.1,13.5,11.9$; IR (thin film) $3433,2963,2928,2877,1724,1657,1436$ ,1381, 1317, 1266, 1204, 1171, 1050, $984 \mathrm{~cm}^{-1}$; LRMS (FAB, M+1) calc'd for $\mathrm{C}_{22} \mathrm{H}_{40} \mathrm{O}_{7} 417.3$, found 417.3.
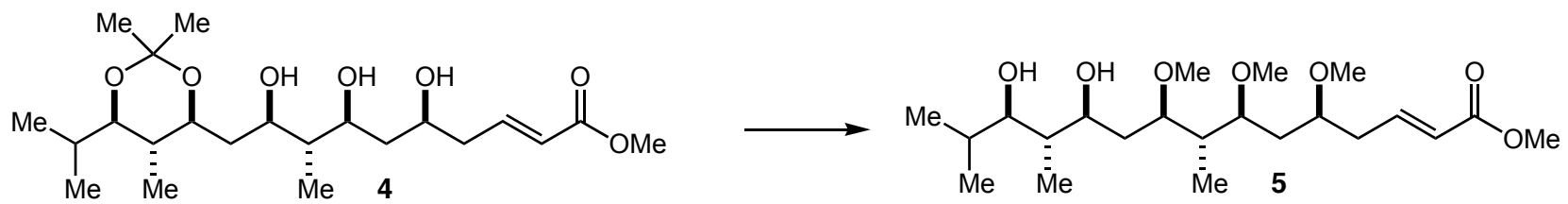

A solution of $4(23 \mathrm{mg}, 0.055 \mathrm{mmol})$ in $1.0 \mathrm{~mL}$ of $\mathrm{CH}_{2} \mathrm{Cl}_{2}$ was added by canula to a suspension of activated $4 \AA$ molecular sieves $(300 \mathrm{mg})$ in $3.0 \mathrm{~mL} \mathrm{CH}_{2} \mathrm{Cl}_{2}$. Proton Sponge $(118 \mathrm{mg}, 0.55 \mathrm{mmol})$ was added as a solid, followed by trimethyloxonium tetrafluoroborate $(73 \mathrm{mg}, 0.495 \mathrm{mmol})$. After 1 hour, the reaction mixture was filtered through a pad of Celite, with 6 x $3.0 \mathrm{~mL}$ of EtOAc washes. The filtrate was washed with $\mathrm{H}_{2} \mathrm{O}$, washed with $1 \mathrm{M}$ aqueous $\mathrm{CuSO}_{4}$, dried over $\mathrm{MgSO}_{4}$, filtered and concentrated. The residue was passed through a plug of silica gel with $5 \%$ EtOAc/Hexanes as the eluting solvent, and the filtrate was concentrated. To a solution of the residue in $5 \mathrm{~mL}$ of THF, was added $2 \mathrm{~mL}$ of $1 \mathrm{~N} \mathrm{HCl}$. After $16 \mathrm{~h}$, the solution was concentrated. The residue was dissolved in EtOAc and washed with saturated aqueous $\mathrm{NaCl}$. The organic phase was dried over $\mathrm{MgSO}_{4}$, filtered and concentrated to afford diol 5 (17 mg, 74\% yield over two steps) as a clear colorless oil. ${ }^{1} \mathrm{H}$ NMR $\left(500 \mathrm{MHz}, \mathrm{CDCl}_{3}\right) \delta$ 7.01-6.96 (m, 1H, $\left.\mathrm{CH}=\mathrm{CHCO}_{2} \mathrm{CH}_{3}\right), 5.91\left(\mathrm{~d}, J=15.7 \mathrm{~Hz}, 1 \mathrm{H}, \mathrm{CH}=\mathrm{CHCO}_{2} \mathrm{CH}_{3}\right), 4.45(\mathrm{~s}$ 1H, OH), 4.40 (s, 1H, OH), 3.81-3.76 (m, 1H, CHOR), 3.73 (s, 3H, $\left.\mathrm{CO}_{2} \mathrm{CH}_{3}\right), 3.71-3.67$ (m, 1H, $\mathrm{CHOCH}_{3}$ ), 
3.50-3.45 (m, 1H, $\left.\mathrm{CHOCH}_{3}\right), 3.42-3.38\left(\mathrm{~m}, 1 \mathrm{H},\left(\mathrm{CH}_{3}\right)_{2} \mathrm{CHCHOH}\right), 3.35-3.33(\mathrm{~m}, 6 \mathrm{H}$, two OCH $) 3.26(\mathrm{~s}, 3 \mathrm{H}$, $\left.\mathrm{OCH}_{3}\right), \quad 3.13-3.08\left(\mathrm{~m}, 1 \mathrm{H}, \mathrm{CHOCH}_{3}\right), 2.57-2.50\left(\mathrm{~m}, 1 \mathrm{H}\right.$, one $\left.\mathrm{CH}_{2} \mathrm{CH}=\mathrm{CHCO}_{2} \mathrm{CH}_{3}\right), 2.45-2.37(\mathrm{~m}, 1 \mathrm{H}$, one $\left.\mathrm{CH}_{2} \mathrm{CH}=\mathrm{CHCO}_{2} \mathrm{CH}_{3}\right), 2.22-2.14\left(\mathrm{~m}, 1 \mathrm{H}, \mathrm{CHCH}_{3}\right), 1.87-1.71\left(\mathrm{~m}, 3 \mathrm{H}, \mathrm{CH}\left(\mathrm{CH}_{3}\right)_{2}\right.$ and two $\mathrm{CH}$ ), 1.70-1.58 (m, $2 \mathrm{H}$, one $\mathrm{CHCH}_{3}$ and one $\left.\mathrm{CH}_{2}\right), 1.54-1.44\left(\mathrm{~m}, 1 \mathrm{H}, \mathrm{CH}_{2}\right), 1.01\left(\mathrm{~d}, J=6.8 \mathrm{~Hz}, 3 \mathrm{H}, \mathrm{CH}_{3}\right), 0.87(\mathrm{~d}, J=6.7 \mathrm{~Hz}, 3 \mathrm{H}$, $\left.\mathrm{CH}_{3}\right), 0.84\left(\mathrm{~d}, J=7.0 \mathrm{~Hz}, 3 \mathrm{H}, \mathrm{CH}_{3}\right), 0.78\left(\mathrm{~d}, J=6.8 \mathrm{~Hz}, 3 \mathrm{H}, \mathrm{CH}_{3}\right) ;{ }^{13} \mathrm{C} \mathrm{NMR}\left(100 \mathrm{MHz}, \mathrm{CDCl}_{3}\right) \delta 166.7$, $145.2,123.3,83.1,79.9,79.6,77.6,76.7,56.7,56.6,51.5,41.7,36.9,36.4,34.8,33.5,33.0,20.4,14.2,13.3$, 10.2; IR (thin film) 3438, 2963, 2931, 2822, 1723, 1659, 1459, 1434, 1376, 1324, 1273, 1196, 1164, 1087,990 $\mathrm{cm}^{-1} ;$ LRMS (FAB, M+1) calc'd for $\mathrm{C}_{22} \mathrm{H}_{42} \mathrm{O}_{7} 419.29$, found 419.28.

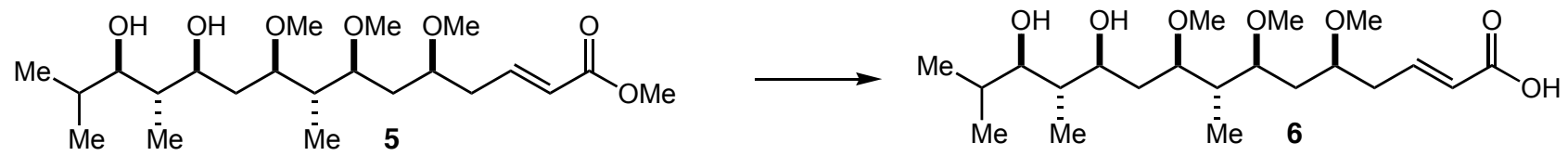

To a solution of ester $5(14.0 \mathrm{mg}, 0.033 \mathrm{mmol})$ in $0.5 \mathrm{~mL}$ of THF and $0.45 \mathrm{~mL}$ of $\mathrm{H}_{2} \mathrm{O}$ was added $50 \mu \mathrm{L}$ of $1 \mathrm{~N} \mathrm{NaOH}$. After 1.5 hours, an additional $25 \mu \mathrm{L}$ of $1 \mathrm{~N} \mathrm{NaOH}$ solution was added. After 6 hours, the pH of the solution was adjusted to $\sim 2$ by the addition of $1 \mathrm{~N} \mathrm{NaHSO}_{4}$. The solution was saturated with $\mathrm{NaCl}$ and extracted three times with EtOAc. The combined organic layers were dried over $\mathrm{MgSO}_{4}$, filtered and concentrated to afford acid 6 (12.0 mg, 90\% yield), which was used without further purification. ${ }^{1} \mathrm{H} \mathrm{NMR}\left(400 \mathrm{MHz}, \mathrm{CDCl}_{3}\right) \delta$ 7.11-7.04 (m, 1H, CH=CHCO $2 \mathrm{H}), 5.91\left(\mathrm{~d}, J=15.6 \mathrm{~Hz}, 1 \mathrm{H}, \mathrm{CH}=\mathrm{CHCO}_{2} \mathrm{H}\right), 3.81-3.77$ (m, 1H, CHOR), 3.73$3.66(\mathrm{~m}, 1 \mathrm{H}, \mathrm{CHOR}), 3.52-3.46(\mathrm{~m}, 1 \mathrm{H}, \mathrm{CHOR}), 3.52-3.46(\mathrm{~m}, 1 \mathrm{H}, \mathrm{CHOR}), 3.41\left(\mathrm{dd}, J_{1}=8.6 \mathrm{~Hz}\right.$ and $J_{2}=2.4$ $\left.\mathrm{Hz}, 1 \mathrm{H},\left(\mathrm{CH}_{3}\right)_{2} \mathrm{CHCHOH}\right), 3.35\left(\mathrm{~s}, 3 \mathrm{H}, \mathrm{OCH}_{3}\right), 3.34\left(\mathrm{~s}, 3 \mathrm{H}, \mathrm{OCH}_{3}\right), 3.26\left(\mathrm{~s}, 3 \mathrm{H}, \mathrm{OCH}_{3}\right), 3.14-3.08(\mathrm{~m}, 1 \mathrm{H}$, CHOR), 2.59-2.51 (m, $1 \mathrm{H}$, one $\left.\mathrm{CH}_{2} \mathrm{CH}=\mathrm{CHCO}_{2} \mathrm{H}\right), 2.49-2.40\left(\mathrm{~m}, 1 \mathrm{H}\right.$, one $\left.\mathrm{CH}_{2} \mathrm{CH}=\mathrm{CHCO}_{2} \mathrm{H}\right), 2.21-2.13(\mathrm{~m}$, $\left.1 \mathrm{H}, \mathrm{CHCH}_{3}\right), 1.84-1.44\left(\mathrm{~m}, 6 \mathrm{H}, \mathrm{CH}\left(\mathrm{CH}_{3}\right)_{2}\right.$ and $\mathrm{CHCH}_{3}$ and two $\left.\mathrm{CH}_{2}\right), 1.00\left(\mathrm{~d}, J=6.9 \mathrm{~Hz}, 3 \mathrm{H}, \mathrm{CH}_{3}\right), 0.87-0.83$ $\left(\mathrm{m}, 6 \mathrm{H}\right.$, two $\left.\mathrm{CH}_{3}\right), 0.78\left(\mathrm{~d}, J=6.8 \mathrm{~Hz}, 3 \mathrm{H}, \mathrm{CH}_{3}\right) ;{ }^{13} \mathrm{C} \mathrm{NMR}\left(100 \mathrm{MHz}, \mathrm{CDCl}_{3}\right) \delta 170.4,147.6,123.1,83.0$, 79.8, 79.6, 77.0, 76.7, 56.7, 56.7, 56.1, 41.6, 36.9, 36.5, 34.7, 33.5, 33.0, 20.4, 14.2, 13.3, 10.2; IR (thin film) 3417, 2962, 2925, 2821, 2620, 1703, 1651, 1457, 1420, 1375, 1263, 1184, $1084 \mathrm{~cm}^{-1} ; \quad$ LRMS (FAB, M+1) calc'd for $\mathrm{C}_{21} \mathrm{H}_{40} \mathrm{O}_{7}$ 405.3, found 406.3. 

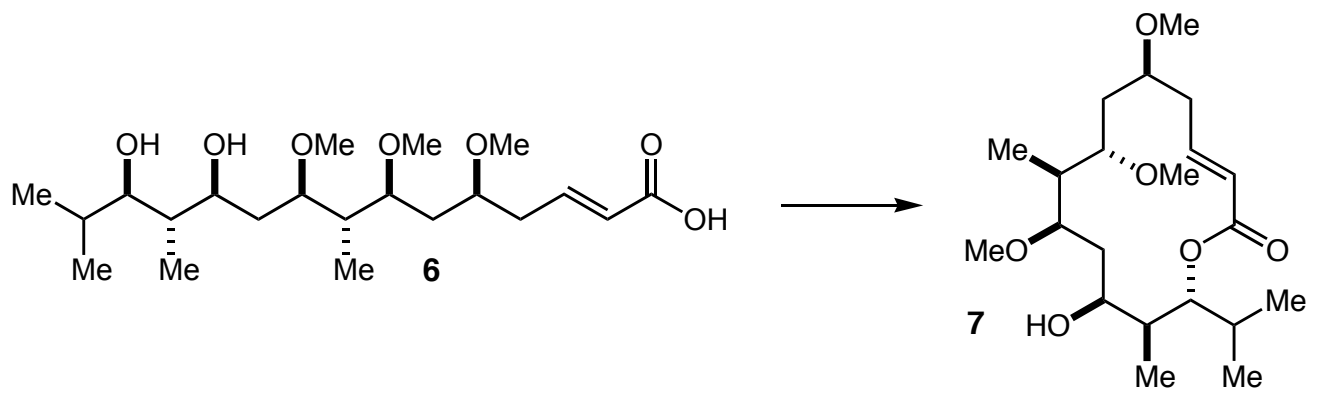

To a solution of acid $6(6.0 \mathrm{mg}, 0.015 \mathrm{mmol})$ in $1.0 \mathrm{~mL}$ of toluene was added $\mathrm{Et}_{3} \mathrm{~N}(10 \mu \mathrm{L}, 0.074 \mathrm{mmol})$. After 20 min a solution of 2,4,6-trichlorobenzoyl chloride $(3.0 \mu \mathrm{L}, 0.019 \mathrm{mmol})$ in $100 \mu \mathrm{L}$ of PhMe was then added. After $1.5 \mathrm{~h}$, the solution was concentrated to a volume of $\sim 300 \mu \mathrm{L}$ with a positive flow of argon and stirred an additional 4 hours. The solution was then diluted with $5.0 \mathrm{~mL}$ of toluene, and 4dimethylaminopyridine $(6.0 \mathrm{mg}, 0.045 \mathrm{mmol})$ was added. After $30 \mathrm{~min}$, the solution was concentrated to $\sim 0.5$ $\mathrm{mL}$ volume and loaded directly onto a silica gel column. Gradient elution with $0 \rightarrow 10 \%$ EtOAc/Hexanes, followed by $20 \rightarrow 25 \%$ EtOAc/Hexanes led to the isolation of 7 ( $4.8 \mathrm{mg}, 84 \%$ yield) as an oil. ${ }^{1} \mathrm{H}$ NMR (500 $\left.\mathrm{MHz}, \mathrm{CDCl}_{3}\right) \delta$ 6 6.82-6.76 (m, 1H, CH=CHCO $\left.2 \mathrm{R}\right), 5.91\left(\mathrm{~d}, J=16.0 \mathrm{~Hz}, 1 \mathrm{H}, \mathrm{CH}=\mathrm{CHCO}_{2} \mathrm{R}\right), 4.72\left(\mathrm{dd}, J_{l}=8.7\right.$ $\mathrm{Hz}$ and $\left.J_{2}=2.5 \mathrm{~Hz}, 1 \mathrm{H},\left(\mathrm{CH}_{3}\right)_{2} \mathrm{CHCHOCOR}\right), 4.08-4.03(\mathrm{~m}, 1 \mathrm{H}, \mathrm{CHOH}), 3.74(\mathrm{~s}, 1 \mathrm{H}, \mathrm{OH}), 3.70-3.64(\mathrm{~m}, 1 \mathrm{H}$, $\left.\mathrm{CHOCH}_{3}\right), 3.49-3.44\left(\mathrm{~m}, 4 \mathrm{H}, \mathrm{CHOCH}_{3}\right.$ and $\left.\mathrm{OCH}_{3}\right), 3.37\left(\mathrm{~s}, 3 \mathrm{H}, \mathrm{OCH}_{3}\right), 3.36\left(\mathrm{~s}, 3 \mathrm{H}, \mathrm{OCH}_{3}\right), 3.12-3.07(\mathrm{~m}, 1 \mathrm{H}$, $\left.\mathrm{CHOCH}_{3}\right), 2.69-2.62\left(\mathrm{~m}, 1 \mathrm{H}\right.$, one $\left.\mathrm{CH}_{2} \mathrm{CH}=\mathrm{CHCO}_{2} \mathrm{R}\right), 2.47-2.40\left(\mathrm{~m}, 1 \mathrm{H}, \mathrm{CHCH}_{3}\right)$ 2.40-2.33 $(\mathrm{m}, 1 \mathrm{H}$, one $\left.\mathrm{CH}_{2} \mathrm{CH}=\mathrm{CHCO}_{2} \mathrm{R}\right), 2.10-2.00\left(\mathrm{~m}, 2 \mathrm{H}, \mathrm{CHCH}_{3}\right.$ and $\left.\mathbf{C H}\left(\mathrm{CH}_{3}\right)_{2}\right), 1.91-1.84\left(\mathrm{~m}, 1 \mathrm{H}, \mathrm{CH}_{2}\right), 1.51-1.47(\mathrm{~m}, 2 \mathrm{H}$, $\left.\mathrm{CH}_{2}\right), 1.33-1.26\left(\mathrm{~m}, 1 \mathrm{H}, \mathbf{C H}_{2}\right), 1.00\left(\mathrm{~d}, J=6.7 \mathrm{~Hz}, 3 \mathrm{H}, \mathbf{C H}_{3}\right), 0.95\left(\mathrm{~d}, J=7.3 \mathrm{~Hz}, 3 \mathrm{H}, \mathrm{CH}_{3}\right), 0.90(\mathrm{~d}, J=6.7 \mathrm{~Hz}$, $\left.3 \mathrm{H}, \mathrm{CH}_{3}\right), 0.87\left(\mathrm{~d}, J=7.2 \mathrm{~Hz}, 3 \mathrm{H}, \mathrm{CH}_{3}\right) ;{ }^{13} \mathrm{C} \mathrm{NMR}\left(75 \mathrm{MHz}, \mathrm{CDCl}_{3}\right) \delta 165.1,143.2,125.4,86.8,81.6,79.2$, 77.2, 72.1, 57.1, 56.6, 56.4, 40.0, 35.6, 34.4, 34.4, 32.4, 30.2, 19.6, 18.8, 13.7, 7.2; IR (thin film) 3503, 2963, 2931, 2879, 2822, 1723, 1646, 1459, 1369, 1247, 1164, 1087 $\mathrm{cm}^{-1}$; LRMS (FAB, M+1) calc'd for $\mathrm{C}_{21} \mathrm{H}_{38} \mathrm{O}_{6}$ 387.27 , found 387.31 .

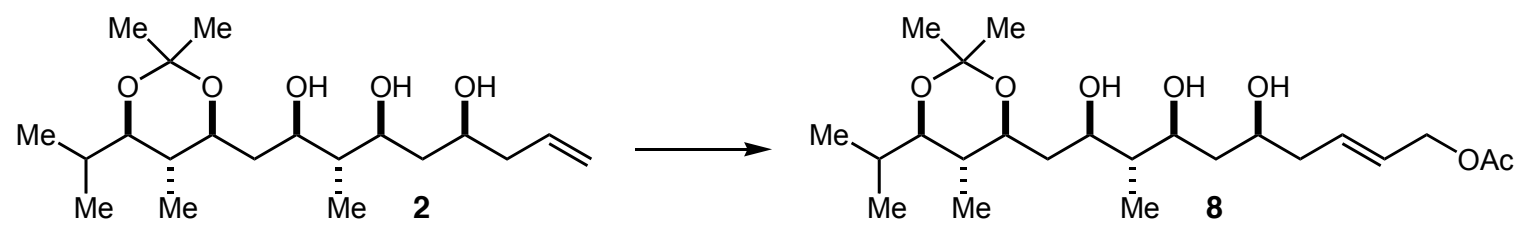

Tricylcohexylphosphine[1,3-bis(2,4,6-trimethylphenyl)-4,5-dihydroimidazol-2-ylidene][benzylidene] ruthenium (IV) dichloride (135 mg, 0.03 equiv.) was weighed out in a glove box, then taken outside the box and 
dissolved in $1.0 \mathrm{~mL}$ of $\mathrm{CH}_{2} \mathrm{Cl}_{2}$. The resulting red solution was added by canula to a solution of 2 (1.13 g, 3.15 mmol) and allyl acetate $(6.8 \mathrm{~mL}, 62.8 \mathrm{mmol})$ in $14 \mathrm{~mL}$ of $\mathrm{CH}_{2} \mathrm{Cl}_{2}$. A reflux condenser was attached to the reaction flask and heating to reflux began. After 48 hours, the reaction mixture was concentrated and the residue was subjected to silica gel chromatography, initially eluting with a slow gradient of $0 \rightarrow 30 \%$ EtOAc/Hexanes in order to separate the impurities (mainly allyl acetate derivatives) and unreacted 2. Increasing the EtOAc concentration to $30 \rightarrow 40 \%$ allowed for the isolation of 8 (950 mg, $70 \%$ yield) as a clear colorless oil. ${ }^{1} \mathrm{H}$ NMR $\left(500 \mathrm{MHz}_{\mathrm{CDCl}}\right) \delta$ 5.89-5.80 (m, 1H, CH=CH), 5.66-5.58 (m, 1H, CH=CH), $4.91(\mathrm{~s}, 1 \mathrm{H}, \mathrm{OH}), 4.55-4.45(\mathrm{~m}$, 3H, $\mathrm{CH}_{2} \mathrm{OAc}$ and $\left.\mathrm{OH}\right), 4.27(\mathrm{~s}, 1 \mathrm{H}, \mathrm{OH}), 3.95-3.86$ (m, 2H, two CHOR), 3.74-3.66 (m, 2H, two CHOR), 3.363.31 (m, 1H, CHOR), 2.32-2.18 (m, 2H, $\left.\mathrm{CH}_{2} \mathrm{CH}=\mathrm{CHCH}_{2} \mathrm{OAc}\right), 2.04$ (s, 3H, $\left.\mathrm{CH}_{2} \mathrm{OCOCH}_{3}\right), 2.04-1.99$ (m, 1H, $\left.\mathrm{CH}_{2}\right), 1.89-1.81\left(\mathrm{~m}, 1 \mathrm{H}, \mathrm{CH}_{2}\right), 1.70-1.65\left(\mathrm{~m}, 1 \mathrm{H}, \mathrm{CH}_{2}\right), 1.58-1.50\left(\mathrm{~m}, 1 \mathrm{H}, \mathrm{CH}_{2}\right), 1.49-1.38\left(\mathrm{~m}, 6 \mathrm{H}\right.$, three $\mathrm{CH}_{2}$ and one $\left.\mathrm{ROC}\left(\mathrm{CH}_{3}\right)_{2} \mathrm{OR}\right), 1.34$ (s, 3H, one $\left.\mathrm{ROC}\left(\mathrm{CH}_{3}\right)_{2} \mathrm{OR}\right), 0.93\left(\mathrm{~d}, J=6.8 \mathrm{~Hz}, 3 \mathrm{H}, \mathrm{CH}_{3}\right), 0.83(\mathrm{~d}, J=6.8 \mathrm{~Hz}$, $\left.3 \mathrm{H}, \mathrm{CH}_{3}\right), 0.78\left(\mathrm{~d}, J=6.8 \mathrm{~Hz}, 3 \mathrm{H}, \mathrm{CH}_{3}\right), 0.74\left(\mathrm{~d}, J=6.5 \mathrm{~Hz}, 3 \mathrm{H}, \mathrm{CH}_{3}\right) ;{ }^{13} \mathrm{C} \mathrm{NMR}\left(75 \mathrm{MHz}, \mathrm{CDCl}_{3}\right) \delta 170.8$ $132.5,126.4,98.1,78.0,76.8,72.1,71.7,71.4,65.1,44.8,40.7,39.7,37.7,36.2,29.9,29.9,28.0,28.0,21.0$, 19.6, 19.6; IR (thin film) 3436, 2968, 2927, 2873, 1741, 1466, 1434, 1245, 1205, 1172, 1052, $1027 \mathrm{~cm}^{-1}$; LRMS (FAB, $\mathrm{M}+1$ ) calc'd for $\mathrm{C}_{23} \mathrm{H}_{42} \mathrm{O}_{7} 431.3$, found 431.6.
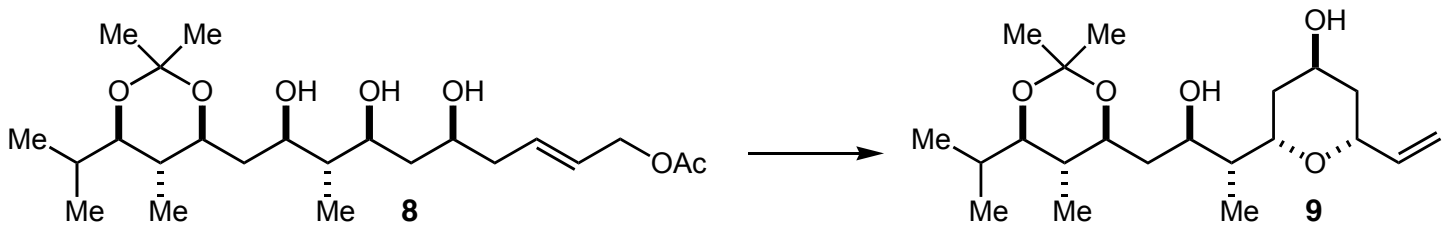

To a solution of $\mathrm{Pd}\left(\mathrm{PPh}_{3}\right)_{4}(50 \mathrm{mg}, 0.04 \mathrm{mmol})$ in $2.0 \mathrm{~mL}$ of toluene was added $\mathrm{Et}_{3} \mathrm{~N}(62 \mu \mathrm{L}, 0.442 \mathrm{mmol})$. A solution of $\mathbf{8}(95 \mathrm{mg}, 0.22 \mathrm{mmol})$ in $1.0 \mathrm{~mL}$ of toluene was then added by cannula. After 5 hours, the reaction was concentrated, and the residue was purified by silica gel chromatography (gradient elution with $5 \rightarrow 15 \%$ EtOAc/Hexanes) to afford 9 (73 mg, 90\% yield). ${ }^{1} \mathrm{H} \mathrm{NMR}\left(500 \mathrm{MHz}, \mathrm{CDCl}_{3}\right) \delta 5.83-5.77\left(\mathrm{~m}, 1 \mathrm{H}, \mathrm{CH}^{2} \mathrm{CH}_{2}\right)$, $5.23\left(\mathrm{ddd}, J_{1}=15.9 \mathrm{~Hz}\right.$ and $\left.J_{2}=J_{3}=1.5 \mathrm{~Hz}, 1 \mathrm{H}, \mathrm{CH}=\mathrm{CH}_{2}\right), 5.06\left(\mathrm{ddd}, J_{1}=10.6 \mathrm{~Hz}\right.$ and $J_{2}=J_{3}=1.5 \mathrm{~Hz}, 1 \mathrm{H}$, $\left.\mathrm{CH}=\mathrm{CH}_{2}\right), 4.31-4.24\left(\mathrm{~m}, 2 \mathrm{H}\right.$, two CHOR), $3.96(\mathrm{~s}, 1 \mathrm{H}, \mathrm{OH}), 3.92-3.85\left(\mathrm{~m}, 2 \mathrm{H}\right.$, two CHOR), 3.68 (ddd, $J_{l}=J_{2}$ $=10.0 \mathrm{~Hz}$ and $\left.J_{3}=2.0 \mathrm{~Hz}, 1 \mathrm{H}, \mathrm{CHOR}\right), 3.33\left(\mathrm{dd}, J_{1}=10.3 \mathrm{~Hz}\right.$ and $\left.J_{2}=2.0 \mathrm{~Hz}, 1 \mathrm{H},\left(\mathrm{CH}_{3}\right)_{2} \mathrm{CHCHOR}\right), 1.96-$ $1.90\left(\mathrm{~m}, 1 \mathrm{H}, \mathrm{CH}_{2}\right), 1.89-1.79\left(\mathrm{~m}, 2 \mathrm{H}, \mathrm{CH}_{2}\right), 1.78-1.68\left(\mathrm{~m}, 2 \mathrm{H}, \mathrm{CH}_{2}\right), 1.56-1.48\left(\mathrm{~m}, 2 \mathrm{H}, \mathrm{CH}_{2}\right), 1.46-1.38(\mathrm{~m}$, 5H, two $\mathrm{CH}_{2}$ and one $\left.\mathrm{ROC}\left(\mathrm{CH}_{3}\right)_{2} \mathrm{OR}\right), 1.34$ (s, 3H, one $\left.\mathrm{ROC}\left(\mathrm{CH}_{3}\right)_{2} \mathrm{OR}\right), 0.93(\mathrm{~d}, J=6.9 \mathrm{~Hz}, 3 \mathrm{H}, \mathrm{CH}), 0.88(\mathrm{~d}$, 
$\left.J=7.0 \mathrm{~Hz}, 3 \mathrm{H}, \mathrm{CH}_{3}\right), 0.83\left(\mathrm{~d}, J=6.9 \mathrm{~Hz}, 3 \mathrm{H}, \mathrm{CH}_{3}\right), 0.73\left(\mathrm{~d}, J=6.6 \mathrm{~Hz}, 3 \mathrm{H}, \mathrm{CH}_{3}\right) ; \quad{ }^{13} \mathrm{C} \mathrm{NMR}(75 \mathrm{MHz}$, $\left.\mathrm{CDCl}_{3}\right) \delta 139.3,114.3,97.9,77.4,76.8,72.4,72.2,64.6,64.4,43.2,43.1,38.4,36.2,35.9,34.9,30.1,30.0$ 28.1, 28.0, 19.6, 19.6; IR (thin film) 3436, 2966, 2927, 2879, 1464, 1418, 1381, 1262, 1204, 1173, $1055 \mathrm{~cm}^{-1}$ LRMS (FAB, M+1) calc'd for $\mathrm{C}_{21} \mathrm{H}_{38} \mathrm{O}_{5} 371.3$, found 371.5 .

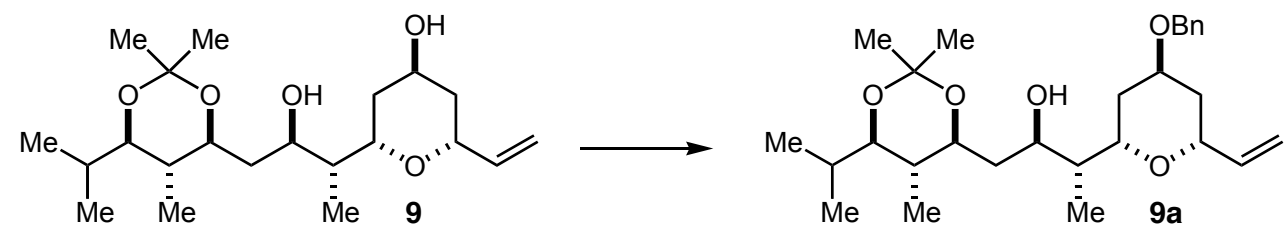

A solution of $9(28 \mathrm{mg}, 0.074 \mathrm{mmol})$ in $3.0 \mathrm{~mL}$ of THF was added by cannula to a suspension of $\mathrm{NaH}(5.7$ $\mathrm{mg}, 0.225 \mathrm{mmol})$ in $1.0 \mathrm{~mL}$ of THF. After $20 \mathrm{~min}$, benzyl bromide (56 $\mu \mathrm{L}, 0.473 \mathrm{mmol})$ was added, followed by $\mathrm{Bu}_{4} \mathrm{NI}(29 \mathrm{mg}, 0.079 \mathrm{mmol})$. The reaction was monitored by thin layer chromatography $(50 \%$ EtOAc/Hexanes), and additional portions of benzyl bromide and $\mathrm{NaH}$ were added until complete conversion was observed. The reaction was quenched with saturated aqueous $\mathrm{NH}_{4} \mathrm{Cl}$, and the aqueous layer was extracted with EtOAc. The combined organic layers were concentrated and the residue purified by silica gel chromatography $\left(0 \rightarrow 20 \%\right.$ EtOAc/Hexanes gradient elution) to afford benzyl ether 9a (31 mg, $91 \%$ yield). ${ }^{1} \mathrm{H}$ NMR (500 MHz, $\left.\mathrm{CDCl}_{3}\right) \delta$ 7.38-7.26 (m, 5H, $\left.\mathrm{C}_{6} \mathbf{H}_{5} \mathrm{CH}_{2} \mathrm{OR}\right), 5.85-5.79\left(\mathrm{~m}, 1 \mathrm{H}, \mathrm{CH}=\mathrm{CH}_{2}\right), 5.22\left(\mathrm{ddd}, J_{1}=17.3\right.$ $\mathrm{Hz}$ and $\left.J_{2}=J_{3}=1.7 \mathrm{~Hz}, 1 \mathrm{H}, \mathrm{CH}=\mathrm{CH}_{2}\right), 5.06\left(\mathrm{ddd}, J_{1}=10.7 \mathrm{~Hz}\right.$ and $\left.J_{2}=J_{3}=1.6 \mathrm{~Hz}, 1 \mathrm{H}, \mathrm{CH}=\mathrm{CH}\right), 4.58(\mathrm{~d}, J=$ $\left.12.2 \mathrm{~Hz}, 1 \mathrm{H}, \mathrm{C}_{6} \mathrm{H}_{5} \mathrm{CH}_{2} \mathrm{OR}\right), 4.55$ (d, $\left.J=12.2 \mathrm{~Hz}, 1 \mathrm{H}, \mathrm{C}_{6} \mathrm{H}_{5} \mathrm{CH}_{2} \mathrm{OR}\right), 4.29-4.22$ (m, 1H, $\left.\mathrm{CH}_{2}=\mathrm{CHCHOR}\right), 4.01-$ 3.96 (m, 1H, CHOR), 3.92-3.89 (m, 1H, CHOR), $3.88(\mathrm{~s}, 1 \mathrm{H}, \mathrm{OH}), 3.82\left(\mathrm{ddd}, J_{l}=10.6 \mathrm{~Hz}\right.$ and $J_{2}=7.4 \mathrm{~Hz}$ and $\left.J_{3}=1.7 \mathrm{~Hz}, 1 \mathrm{H}, \mathrm{CHOR}\right), 3.68\left(\mathrm{ddd}, J_{1}=9.9 \mathrm{~Hz}\right.$ and $\left.J_{2}=J_{3}=2.1 \mathrm{~Hz}, 1 \mathrm{H}, \mathrm{CHOR}\right), 3.34\left(\mathrm{dd}, J_{1}=10.3 \mathrm{~Hz}\right.$ and $J_{2}$ $\left.=2.1 \mathrm{~Hz}, 1 \mathrm{H},\left(\mathrm{CH}_{3}\right)_{2} \mathrm{CHCHOR}\right), 2.03-1.96\left(\mathrm{~m}, 1 \mathrm{H}, \mathrm{CH}_{2}\right), 1.93-1.78\left(\mathrm{~m}, 4 \mathrm{H}, \mathrm{CH}_{2}\right), 1.48-1.39\left(\mathrm{~m}, 7 \mathrm{H}\right.$, four $\mathrm{CH}_{2}$ and one $\left.\mathrm{ROC}\left(\mathrm{CH}_{3}\right)_{2} \mathrm{OR}\right), 1.36$ (s, 3H, one $\left.\mathrm{ROC}\left(\mathrm{CH}_{3}\right)_{2} \mathrm{OR}\right), 0.94\left(\mathrm{~d}, J=6.9 \mathrm{~Hz}, 3 \mathrm{H}, \mathrm{CH}_{3}\right), 0.89$ (d, J = 7.0 Hz, $\left.3 \mathrm{H}, \mathrm{CH}_{3}\right), 0.84\left(\mathrm{~d}, J=6.9 \mathrm{~Hz}, 3 \mathrm{H}, \mathrm{CH}_{3}\right), 0.73\left(\mathrm{~d}, J=6.6 \mathrm{~Hz}, 3 \mathrm{H}, \mathrm{CH}_{3}\right) ;{ }^{13} \mathrm{C} \mathrm{NMR}\left(75 \mathrm{MHz}, \mathrm{CDCl}_{3}\right) \delta 139.4$, $138.9,128.4,127.5,114.2,97.9,77.6,76.6,73.6,73.1,72.7,71.3,70.1,43.2,36.2,35.8,35.7,32.4,30.1,28.1$, 20.0, 19.7, 14.2, 11.9, 11.0; IR (thin film) 3513, 2964, 2926, 2870, 1452, 1421, 1377, 1346, 1302, 1259, 1202, $1171,1059 \mathrm{~cm}^{-1}$; LRMS (FAB, M+1) calc'd for $\mathrm{C}_{28} \mathrm{H}_{44} \mathrm{O}_{5} 461.3$, found 460.5 . 


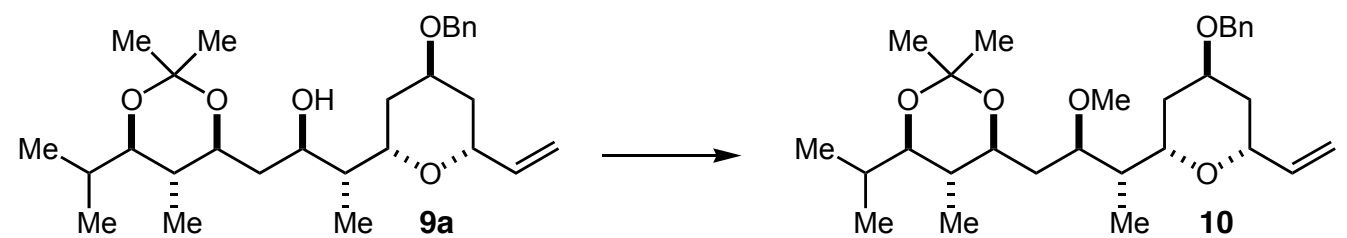

A solution of $9 \mathbf{a}(160 \mathrm{mg}, 0.348 \mathrm{mmol})$ in $1.0 \mathrm{~mL}$ of $\mathrm{CH}_{2} \mathrm{Cl}_{2}$ was added by cannula to a suspension of activated $4 \AA$ molecular sieves $(2.0 \mathrm{~g})$ in $4.0 \mathrm{~mL} \mathrm{CH}_{2} \mathrm{Cl}_{2}$. Proton Sponge (450 mg, $\left.2.09 \mathrm{mmol}\right)$ was added, followed by trimethyloxonium tetrafluoroborate $(240 \mathrm{mg}, 1.63 \mathrm{mmol})$. After $6 \mathrm{~h}$, the reaction mixture was filtered through a pad of Celite, with EtOAc washes. The filtrate was washed with $\mathrm{H}_{2} \mathrm{O}$, washed with $1 \mathrm{~N}$ aqueous $\mathrm{HCl}$, and washed with saturated aqueous $\mathrm{CuSO}_{4}$. The combined organic layers were washed with saturated aqueous $\mathrm{NaCl}$, dried over $\mathrm{MgSO}_{4}$, filtered and concentrated. The residue was purified by silica gel chromatography with $0 \rightarrow 2 \%$ EtOAc/Hexanes to afford 10 (140 mg, 85\% yield). ${ }^{1} \mathrm{H}$ NMR (400 $\left.\mathrm{MHz} \mathrm{CDCl}{ }_{3}\right) \delta$ 7.36-7.26 (m, 5H, $\left.\mathrm{C}_{6} \mathbf{H}_{5} \mathrm{CH}_{2} \mathrm{OR}\right)$, 5.86-5.78 (m, $\left.1 \mathrm{H}, \mathrm{CH}=\mathrm{CH}_{2}\right)$, 5.23-5.17 (m, 1H, $\mathrm{CH}=\mathrm{CH}$ ), 5.09-5.04 (m, $1 \mathrm{H}$, $\left.\mathrm{CH}=\mathrm{CH}_{2}\right), 4.58-4.52\left(\mathrm{~m}, 2 \mathrm{H}, \mathrm{C}_{6} \mathrm{H}_{5} \mathrm{CH}_{2} \mathrm{OR}\right), 4.27-4.21\left(\mathrm{~m}, 1 \mathrm{H}, \mathrm{CH}_{2}=\mathrm{CHCHOR}\right), 3.92-3.88$ (m, 1H, CHOR), 3.75-3.69 (m, 1H, CHOR), 3.63-3.57 (m, 1H, CHOR), 3.53-3.48 (m, 1H, CHOR), 3.33-3.28 (m, 4H, OCH and $^{2}$ $\left.\left(\mathrm{CH}_{3}\right)_{2} \mathrm{CHCHOR}\right), 1.99-1.78\left(\mathrm{~m}, 5 \mathrm{H}, \mathrm{CH}_{2}\right), 1.52-1.30\left(\mathrm{~m}, 10 \mathrm{H}\right.$, four $\mathrm{CH}_{2}$ and $\left.\mathrm{ROC}\left(\mathrm{CH}_{3}\right)_{2} \mathrm{OR}\right), 0.94(\mathrm{~d}, J=6.9$ $\left.\mathrm{Hz}, 3 \mathrm{H}, \mathrm{CH}_{3}\right), 0.84\left(\mathrm{~d}, J=6.8 \mathrm{~Hz}, 3 \mathrm{H}, \mathrm{CH}_{3}\right), 0.83\left(\mathrm{~d}, J=7.0 \mathrm{~Hz}, 3 \mathrm{H}, \mathrm{CH}_{3}\right), 0.74\left(\mathrm{~d}, J=6.6 \mathrm{~Hz}, 3 \mathrm{H}, \mathrm{CH}_{3}\right) ;{ }^{13} \mathrm{C}$ $\operatorname{NMR}\left(100 \mathrm{MHz}, \mathrm{CDCl}_{3}\right) \delta 139.2,138.7,129.1,127.3,127.2$ 113.7, 97.5, 79.3, 77.8, 73.0, 72.8, 72.6, 71.4, $70.1,56.8,40.5,36.8,34.9,32.9,30.3,28.3,20.3,19.8,14.5,12.2,10.3$; IR (thin film) $2965,2934,2876,1456$, 1379, 1261, 1202, 1176, 1094, 1064, $1018 \mathrm{~cm}^{-1}$; LRMS (FAB, M+1) calc'd for $\mathrm{C}_{29} \mathrm{H}_{46} \mathrm{O}_{5}$ 475.3, found 475.4.

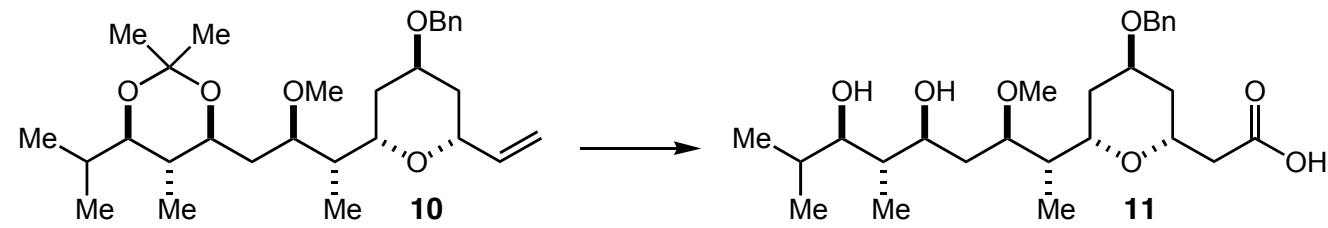

To a cooled $\left(-10^{\circ} \mathrm{C}\right)$ solution of $\mathbf{1 0}(95 \mathrm{mg}, 0.20 \mathrm{mmol})$ in $4.0 \mathrm{~mL} \mathrm{Et}{ }_{2} \mathrm{O}$ was added $\mathrm{BH}_{3} \cdot \mathrm{SMe}_{2}(16 \mu \mathrm{L}, 0.157$ mmol). After 15 minutes the cooling bath was removed and the reaction was stirred at room temperature for an additional 2 hours. $1.5 \mathrm{~mL}$ of a 1:1 EtOH:1N NaOH was then added, followed by $1.0 \mathrm{~mL}$ of $30 \%$ aqueous $\mathrm{H}_{2} \mathrm{O}_{2}$. After $12 \mathrm{~h}, 1.0 \mathrm{~mL}$ of saturated aqueous $\mathrm{NH}_{4} \mathrm{Cl}$ was added, and the biphasic mixture was extracted with EtOAc. The organic layer was washed with saturated aqueous $\mathrm{NaCl}$, dried over $\mathrm{MgSO}_{4}$, filtered and concentrated. Silica gel chromatography with a slow gradient elution (0 to $9 \% \mathrm{EtOAc/Hexanes)} \mathrm{allowed} \mathrm{for} \mathrm{the} \mathrm{isolation} \mathrm{of} \mathbf{1 0 a}$. 
To a suspension of NMO $(20 \mathrm{mg}, 0.168 \mathrm{mmol})$ and activated $4 \AA$ molecular sieves $(400 \mathrm{mg})$ in $3.0 \mathrm{~mL}$ of $\mathrm{CH}_{2} \mathrm{Cl}_{2}$ was added a solution of $\mathbf{1 0 a}(49 \mathrm{mg}, 0.084 \mathrm{mmol})$ in $1.0 \mathrm{~mL}$ of $\mathrm{CH}_{2} \mathrm{Cl}_{2}$ by cannula. To this solution was added $\left(\mathrm{Pr}_{4} \mathrm{~N}\right) \mathrm{RuO}_{4}(6 \mathrm{mg}, 0.017 \mathrm{mmol})$. After 3 hours, the reaction mixture was concentrated and the residue was purified by silica gel chromatography eluting with $4 \%$ EtOAc/Hexanes to afford aldehyde $\mathbf{1 0 b}$ as judged by ${ }^{1} \mathrm{H}$ NMR: $\left(300 \mathrm{MHz}, \mathrm{CDCl}_{3}\right) \delta 9.77\left(\mathrm{dd}, J_{1}=J_{2}=2.4 \mathrm{~Hz}\right)$. To a solution of $\mathbf{1 0 b}$ in $3.0 \mathrm{~mL}$ of THF was added $\mathrm{SMe}_{2}(200 \mu \mathrm{L})$. A solution of $\mathrm{NaClO}_{2}(30 \mathrm{mg}, 0.31 \mathrm{mmol})$ and $\mathrm{NaH}_{2} \mathrm{PO}_{4}(100 \mathrm{mg})$ in $1.2 \mathrm{~mL}$ of $\mathrm{H}_{2} \mathrm{O}$ was added, followed by vigorous stirring for 14 hours. The reaction was then diluted with EtOAc and the phases were separated. The organic layer was washed twice with saturated aqueous $\mathrm{NaCl}$, and the aqueous layer was extracted with EtOAc. The combined organic layers were dried over $\mathrm{MgSO}_{4}$, filtered and concentrated to afford acid 10c, which was used without further purification. The residue (10c) was dissolved in $1.2 \mathrm{~mL}$ of 5:1 AcOH: $\mathrm{H}_{2} \mathrm{O}$, and the resulting solution was heated to $60{ }^{\circ} \mathrm{C}$ for 3 hours. Toluene $(3 \mathrm{~mL})$ was added, and the biphasic solution was concentrated. The residue was dissolved in EtOAc and washed with $\mathrm{H}_{2} \mathrm{O}$, followed by saturated aqueous $\mathrm{NaCl}$. The aqueous layer was extracted with EtOAc, and the combined organic layers were dried over $\mathrm{MgSO}_{4}$, filtered and concentrated to afford 11 (42 mg, 45\% yield over four steps). ${ }^{1} \mathrm{H} \mathrm{NMR}\left(400 \mathrm{MHz}, \mathrm{CDCl}_{3}\right.$ ) $\delta$ 7.37-7.26 (m, 5H, $\left.\mathrm{C}_{6} \mathbf{H}_{5} \mathrm{CH}_{2} \mathrm{OR}\right), 4.59\left(\mathrm{~d}, J=12.2 \mathrm{~Hz}, 1 \mathrm{H}, \mathrm{C}_{6} \mathrm{H}_{5} \mathrm{CH}_{2} \mathrm{OR}\right), 4.53(\mathrm{~d}, J=12.2 \mathrm{~Hz}, 1 \mathrm{H}$, $\mathrm{C}_{6} \mathrm{H}_{5} \mathrm{CH}_{2} \mathrm{OR}$ ), 4.23-4.10 (m, 2H, CHOR), 3.94-3.88 (m, 2H, CHOR), 3.49-3.40 (m, 1H, CHOR), 3.32 (s, 3H, $\left.\mathrm{OCH}_{3}\right), 3.26\left(\mathrm{dd}, J_{1}=10.6 \mathrm{~Hz}\right.$ and $\left.J_{2}=2.0 \mathrm{~Hz}, 1 \mathrm{H},\left(\mathrm{CH}_{3}\right)_{2} \mathrm{CHCHOR}\right), 2.41-2.31\left(\mathrm{~m}, 2 \mathrm{H}, \mathrm{CH}_{2} \mathrm{CO}_{2} \mathrm{H}\right), 2.08-1.95$ $\left(\mathrm{m}, 2 \mathrm{H}, \mathrm{CH}_{2}\right), 1.88-1.78\left(\mathrm{~m}, 3 \mathrm{H}, \mathrm{CH}_{2}\right), 1.44-1.26\left(\mathrm{~m}, 5 \mathrm{H}, \mathrm{CH}_{2}\right), 1.00(\mathrm{~d}, J=6.8 \mathrm{~Hz}, 3 \mathrm{H}, \mathrm{CH}), 0.83(\mathrm{~d}, J=6.8$ $\left.\mathrm{Hz}, 3 \mathrm{H}, \mathrm{CH}_{3}\right), 0.82\left(\mathrm{~d}, J=6.9 \mathrm{~Hz}, 3 \mathrm{H}, \mathrm{CH}_{3}\right), 0.76\left(\mathrm{~d}, J=7.0 \mathrm{~Hz}, 3 \mathrm{H}, \mathrm{CH}_{3}\right) ;{ }^{13} \mathrm{C} \mathrm{NMR}\left(100 \mathrm{MHz} \mathrm{CDCl}_{3}\right) \delta$ $173.2,138.4,129.5,128.3,127.5,127.3,82.4,74.3,72.6,71.3,70.5,70.1,55.8,42.2,41.6,38.5,35.1,34.8$, 29.3, 27.4, 20.5, 13.5, 10.4, 9.1; IR (thin film) 3410, 2964, 2635, 1717, 1541, 1457, 1420, 1378, 1337, 1269, $1064 \mathrm{~cm}^{-1}$; LRMS (FAB, M+1) calc'd for $\mathrm{C}_{26} \mathrm{H}_{42} \mathrm{O}_{7} 467.3$, found 467.2.

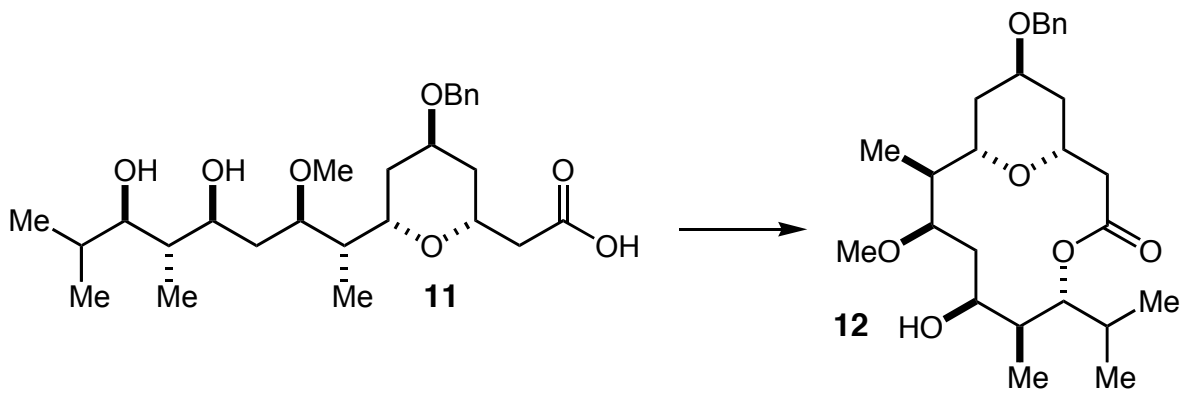


To a solution of $11(3.1 \mathrm{mg}, 0.0064 \mathrm{mmol})$ in $1.0 \mathrm{~mL}$ of toluene was added $\mathrm{Et}_{3} \mathrm{~N}(5 \mu \mathrm{L}, 0.037 \mathrm{mmol})$. A solution of 2,4,6-trichlorobenzoyl chloride $(1.4 \mu \mathrm{L}, 0.0089 \mathrm{mmol})$ in $100 \mu \mathrm{L}$ of toluene was then added. After $1.5 \mathrm{~h}$, the solution was then concentrated to a volume of $\sim 300 \mu \mathrm{L}$ with a positive flow of argon and stirred an additional 4 hours. Toluene $(4.0 \mathrm{~mL})$ was added and the resulting solution was added dropwise by cannula to a solution of 4-dimethylaminopyridine $(6.0 \mathrm{mg}, 0.045 \mathrm{mmol})$ in $30.0 \mathrm{~mL}$ of PhMe. After 1.5 hours, the reaction mixture was washed with $10 \mathrm{~mL}$ of $\mathrm{H}_{2} \mathrm{O}, 10 \mathrm{~mL}$ of $0.1 \mathrm{M}$ aqueous $\mathrm{NaHSO}_{4}$, saturated aqueous $\mathrm{NaCl}$, dried over $\mathrm{MgSO}_{4}$, filtered and concentrated. Purification of the residue by silica gel chromatography, with slow gradient elution $5 \rightarrow 15 \%$ EtOAc/Hexanes afforded 12 (1.5 mg, 50\% yield) as a white solid. ${ }^{1} \mathrm{H}$ NMR (500 $\left.\mathrm{MHz} \mathrm{CDCl}_{3}\right)$ $\delta$ 7.35-7.33 (m, 4H, $\left.\mathrm{C}_{6} \mathbf{H}_{5} \mathrm{CH}_{2} \mathrm{OR}\right), 7.30-7.26\left(\mathrm{~m}, 1 \mathrm{H}, \mathrm{C}_{6} \mathbf{H}_{5} \mathrm{CH}_{2} \mathrm{OR}\right), 4.73\left(\mathrm{dd}, J_{1}=9.8 \mathrm{~Hz}\right.$ and $J_{2}=2.7 \mathrm{~Hz}, 1 \mathrm{H}$, $\left.\left(\mathrm{CH}_{3}\right)_{2} \mathrm{CHCHOCOR}\right), 4.53$ (s, 2H, $\left.\mathrm{C}_{6} \mathrm{H}_{5} \mathrm{CH}_{2} \mathrm{OR}\right), 4.35-4.28$ (m, 1H, CHOR), 4.21-4.13 (m, 1H, CHOR), 3.873.82 (m, 2H, CHOR), 3.33 (s, 3H, OCH $), 2.34-2.27$ (m, 2H, $\left.\mathrm{CH}_{2} \mathrm{CO}_{2} \mathrm{H}\right), 2.18-2.12$ (m, 1H, CH ), 1.99-1.84 (m, 4H, CH$), 1.74-1.66\left(\mathrm{~m}, 1 \mathrm{H}, \mathrm{CH}_{2}\right), 1.44-1.25\left(\mathrm{~m}, 3 \mathrm{H}, \mathrm{CH}_{2}\right), 0.94(\mathrm{~d}, J=6.9 \mathrm{~Hz}, 3 \mathrm{H}, \mathrm{CH}), 0.92(\mathrm{~d}, J=6.9$ $\left.\mathrm{Hz}, 3 \mathrm{H}, \mathbf{C H}_{3}\right), 0.88$ (d, $\left.J=7.1 \mathrm{~Hz}, 3 \mathrm{H}, \mathrm{CH}_{3}\right), 0.88\left(\mathrm{~d}, J=6.8 \mathrm{~Hz}, 3 \mathrm{H}, \mathrm{CH}_{3}\right) ;{ }^{13} \mathrm{C} \mathrm{NMR}\left(100 \mathrm{MHz} \mathrm{CDCl}_{3}\right) \delta$ $172.3,138.6,128.2,127.5,127.2,83.2,79.4,72.4,71.3,71.0,70.2,69.5,57.7,42.4,39.8,38.7,35.7,34.3,30.6$, 30.0. 20.5, 15.7, 14.7, 10.9; IR (thin film) 3410, 2965, 2920, 1729, 1466, 1446, 1378, 1330, 1263, 1093,1059 $\mathrm{cm}^{-1}$; LRMS (FAB, M+1) calc'd for $\mathrm{C}_{26} \mathrm{H}_{40} \mathrm{O}_{6} 449.3$, found 449.6.

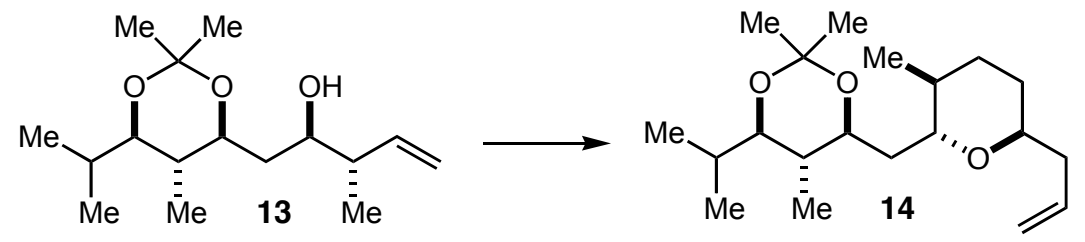

A solution of 13 (340 mg, $1.26 \mathrm{mmol})$ in $12.0 \mathrm{~mL}$ THF was added by cannula to an oven-dried $45 \mathrm{~mL}$ capacity Parr high pressure reaction apparatus. $\mathrm{Rh}(\mathrm{acac})(\mathrm{CO})_{2}(13 \mathrm{mg}, 0.05 \mathrm{mmol})$ was added, followed by $\mathrm{PPh}_{3}(40 \mathrm{mg}, 0.151 \mathrm{mmol})$. The reaction vessel was sparged twice with $1: 1 \mathrm{H}_{2}: \mathrm{CO}$, then charged to 400 psi of 1:1 $\mathrm{H}_{2}: \mathrm{CO}$ and heated to $55^{\circ} \mathrm{C}$ (oil bath temp.) for 22 hours. The reaction apparatus was cooled, vented and the solution was concentrated. To a solution of the residue in $8.0 \mathrm{~mL}$ of $\mathrm{CH}_{2} \mathrm{Cl}_{2}$ was added $\mathrm{Et}_{3} \mathrm{~N}(700 \mu \mathrm{L}, 5.04$ mmol), and 4-dimethylaminopyridine ( $8 \mathrm{mg}, 0.063 \mathrm{mmol})$. Acetic anhydride $(415 \mu \mathrm{L}, 4.41 \mathrm{mmol})$ was then added. After $18 \mathrm{~h}$, the solution was diluted with $\mathrm{CH}_{2} \mathrm{Cl}_{2}$ and washed with saturated aqueous $\mathrm{NH}_{4} \mathrm{Cl}$, saturated aqueous $\mathrm{CuSO}_{4}$, then saturated aqueous $\mathrm{NaCl}$. The organic phase was dried over $\mathrm{MgSO}_{4}$, filtered and 
concentrated. To a cooled $\left(-78^{\circ} \mathrm{C}\right)$ solution of the residue in $7.0 \mathrm{~mL}$ of $\mathrm{CH}_{2} \mathrm{Cl}_{2}$ was added allyltrimethylsilane (400 $\mu \mathrm{L}, 2.52 \mathrm{mmol})$. In a separate flask, a solution of $\mathrm{TiCl}_{4}(225 \mu \mathrm{L}, 1.26 \mathrm{mmol})$ and $\mathrm{Ti}\left(\mathrm{O}^{i} \mathrm{Pr}\right)_{4}(372 \mu \mathrm{L}, 1.26$ mmol) in $4 \mathrm{~mL}$ of $\mathrm{CH}_{2} \mathrm{Cl}_{2}$ was prepared and added by cannula to the reaction mixture. After $1 \mathrm{~h}, 1.0 \mathrm{~mL} \mathrm{of} \mathrm{Et}_{3} \mathrm{~N}$ was added, followed by saturated aqueous $\mathrm{NH}_{4} \mathrm{Cl}$. The mixture was filtered through a pad of Celite. The resulting biphasic mixture was partitioned and the aqueous layer was extracted with $\mathrm{CH}_{2} \mathrm{Cl}_{2}$ and the combined organic layers were washed with saturated aqueous $\mathrm{NaCl}$, dried over $\mathrm{MgSO}_{4}$, filtered, and concentrated. Purification of the residue by silica gel chromatography $(0 \rightarrow 2 \%$ EtOAc/Hexanes) afforded 14 (267 mg, $65 \%$ over three steps) as a clear, colorless oil. ${ }^{1} \mathrm{H}$ NMR $\left(500 \mathrm{MHz}, \mathrm{CDCl}_{3}\right) \delta 5.90-5.82\left(\mathrm{~m}, 1 \mathrm{H}, \mathrm{CH}=\mathrm{CH}_{2}\right), 5.12-5.03$ (m, 2H, $\left.\mathrm{CH}=\mathrm{CH}_{2}\right), 3.85-3.78\left(\mathrm{~m}, 1 \mathrm{H}, \mathrm{CH}_{2}=\mathrm{CHCH}_{2} \mathrm{CHOR}\right), 3.61-3.54(\mathrm{~m}, 2 \mathrm{H}, \mathrm{CHOR}), 3.32\left(\mathrm{dd}, J_{1}=10.2 \mathrm{~Hz}\right.$ and $\left.J_{2}=2.1 \mathrm{~Hz}, 1 \mathrm{H},\left(\mathrm{CH}_{3}\right)_{2} \mathrm{CHCHOR}\right), 2.48-2.41\left(\mathrm{~m}, 1 \mathrm{H}, \mathrm{CH}_{2} \mathrm{CH}=\mathrm{CH}_{2}\right), 2.23-2.14\left(\mathrm{~m}, 1 \mathrm{H}, \mathrm{CH}_{2} \mathrm{CH}_{=} \mathrm{CH}_{2}\right)$, 1.91-1.84 (m, 2H, $\left.\mathbf{C H}_{2}\right), 1.77-1.59\left(\mathrm{~m}, 2 \mathrm{H}, \mathrm{CH}_{2}\right), 1.65-1.58\left(\mathrm{~m}, 1 \mathrm{H},\left(\mathrm{CH}_{3}\right)_{2} \mathrm{CH}\right), 1.57-1.46\left(\mathrm{~m}, 3 \mathrm{H}, \mathrm{CHCH}_{3}\right.$ and two $\left.\mathrm{CH}_{2}\right), 1.40-1.35$ (m, 4H, one $\mathrm{CH}_{2}$ and one $\left.\mathrm{ROC}\left(\mathrm{CH}_{3}\right)_{2} \mathrm{OR}\right), 1.35\left(\mathrm{~s}, 3 \mathrm{H}\right.$, one $\left.\mathrm{ROC}(\mathrm{CH})_{2} \mathrm{OR}\right), 1.01(\mathrm{~d}, J=$ $\left.6.8 \mathrm{~Hz}, 3 \mathrm{H}, \mathrm{CH}_{3}\right), 0.96\left(\mathrm{~d}, J=6.9 \mathrm{~Hz}, 3 \mathrm{H}, \mathrm{CH}_{3}\right), 0.86\left(\mathrm{~d}, J=6.9 \mathrm{~Hz}, 3 \mathrm{H}, \mathrm{CH}_{3}\right), 0.77\left(\mathrm{~d}, J=6.6 \mathrm{~Hz}, 3 \mathrm{H}, \mathrm{CH}_{3}\right)$; ${ }^{13} \mathrm{C} \mathrm{NMR}\left(100 \mathrm{MHz}, \mathrm{CDCl}_{3}\right) \delta 135.8,116.2,97.6,77.9,74.6,72.2,70.6,37.9,36.9,36.2,32.6,30.1,28.1,26.9$, $26.1,20.1,19.5,18.4,14.3,12.0 ;$ IR (thin film) 2955, 2929, 2855, 1648, 1461, 1373, 1259, 1199, $1172 \mathrm{~cm}^{-1}$; LRMS (FAB, M+1) calc'd for $\mathrm{C}_{20} \mathrm{H}_{36} \mathrm{O}_{3} 325.3$, found 325.1.

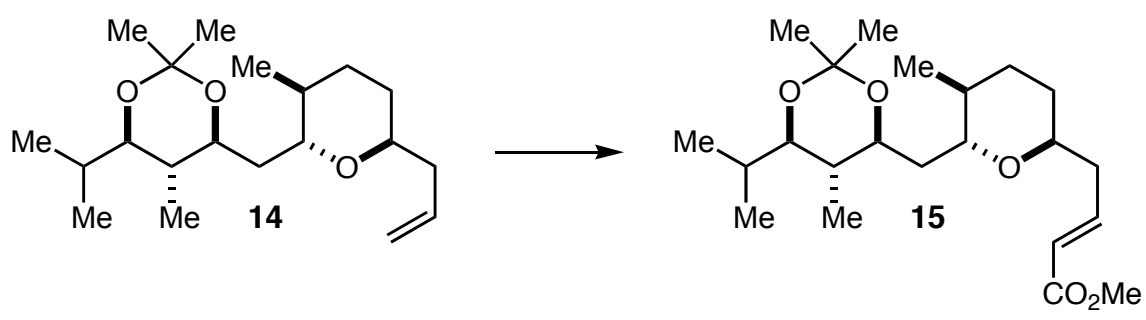

Tricylcohexylphosphine[1,3-bis(2,4,6-trimethylphenyl)-4,5-dihydroimidazol-2-ylidene][benzylidene] ruthenium (IV) dichloride (30 mg, $0.039 \mathrm{mmol}$ ) was weighed out in a glove box, then taken outside the box and dissolved in $1.0 \mathrm{~mL}$ of $\mathrm{CH}_{2} \mathrm{Cl}_{2}$. The resulting red solution was added by cannula to a solution of 14 (255 mg, $0.787 \mathrm{mmol})$ and methyl acrylate $(2.5 \mathrm{~mL}, 27.6 \mathrm{mmol})$ in $7.0 \mathrm{~mL}$ of $\mathrm{CH}_{2} \mathrm{Cl}_{2}$. A reflux condenser was attached to the reaction flask and heating to reflux began. After 20 hours, the solution was concentrated and the residue was purified by silica gel chromatography, initially eluting with a slow gradient of $0 \rightarrow 20 \%$ EtOAc/Hexanes in order to separate the major impurities (mainly methyl acrylate derivatives). Increasing the EtOAc concentration to 
$25 \rightarrow 30 \%$ allowed for the isolation of $\mathbf{1 5}\left(242 \mathrm{mg}, 80 \%\right.$ yield) as a clear colorless oil. ${ }^{1} \mathrm{H}$ NMR (400 MHz, $\left.\mathrm{CDCl}_{3}\right) \delta 7.00-6.93\left(\mathrm{~m}, 1 \mathrm{H}, \mathrm{CH}=\mathrm{CHCO}_{2} \mathrm{CH}_{3}\right), 5.88\left(\mathrm{ddd}, J_{1}=15.7 \mathrm{~Hz}\right.$ and $J_{2}=J_{3}=1.4 \mathrm{~Hz}, 1 \mathrm{H}$, $\left.\mathrm{CH}=\mathrm{CHCO}_{2} \mathrm{CH}_{3}\right), 3.94-3.87\left(\mathrm{~m}, 1 \mathrm{H}, \mathrm{CH}=\mathrm{CHCH}_{2} \mathrm{CHOR}\right), 3.71\left(\mathrm{~s}, 3 \mathrm{H}, \mathrm{CO}_{2} \mathrm{CH}_{3}\right), 3.57-3.49$ (m, 2H, $\left.\mathrm{CHOR}\right)$, $3.28\left(\mathrm{dd}, J_{1}=10.2 \mathrm{~Hz}\right.$ and $\left.J_{2}=2.1 \mathrm{~Hz}, 1 \mathrm{H},\left(\mathrm{CH}_{3}\right)_{2} \mathrm{CHCHOR}\right), 2.60-2.51\left(\mathrm{~m}, 1 \mathrm{H}, \mathrm{CH}_{2} \mathrm{CH}=\mathrm{CHCO}_{2} \mathrm{CH}_{3}\right), 2.33-$ $2.25\left(\mathrm{~m}, 1 \mathrm{H}, \mathrm{CH}_{2} \mathrm{CH}=\mathrm{CHCO}_{2} \mathrm{CH}_{3}\right), 1.89-1.81\left(\mathrm{~m}, 2 \mathrm{H}, \mathrm{CH}_{2}\right), 1.74-1.65\left(\mathrm{~m}, 2 \mathrm{H}, \mathrm{CH}_{2}\right), 1.65-1.57(\mathrm{~m}, 1 \mathrm{H}$, $\left.\left(\mathrm{CH}_{3}\right)_{2} \mathrm{CH}\right), 1.54-1.40\left(\mathrm{~m}, 3 \mathrm{H}, \mathrm{CHCH}_{3}\right.$ and two $\left.\mathrm{CH}_{2}\right), 1.37-1.32\left(\mathrm{~m}, 7 \mathrm{H}\right.$, one $\mathrm{CH}_{2}$ and $\left.\mathrm{ROC}(\mathrm{CH})_{2} \mathrm{OR}\right), 0.99(\mathrm{~d}$, $\left.J=6.8 \mathrm{~Hz}, 3 \mathrm{H}, \mathrm{CH}_{3}\right), 0.93\left(\mathrm{~d}, J=6.9 \mathrm{~Hz}, 3 \mathrm{H}, \mathrm{CH}_{3}\right), 0.83\left(\mathrm{~d}, J=6.8 \mathrm{~Hz}, 3 \mathrm{H}, \mathrm{CH}_{3}\right), 0.74(\mathrm{~d}, J=6.6 \mathrm{~Hz}, 3 \mathrm{H}$, $\left.\mathrm{CH}_{3}\right) ;{ }^{13} \mathrm{C} \mathrm{NMR}\left(100 \mathrm{MHz}, \mathrm{CDCl}_{3}\right) \delta 166.8,146.3,122.6,97.6,77.8,74.8,72.0,69.9,51.4,36.9,36.3,36.2$, 32.4, 30.1, 28.1, 27.1, 26.0, 20.1, 19.4, 18.4, 14.3, 11.9; IR (thin film) 2962, 2929, 2875, 1728, 1661, 1460, 1436, 1379, 1261, 1201, 1174, $1044 \mathrm{~cm}^{-1}$; LRMS (FAB, M+1) calc'd for $\mathrm{C}_{22} \mathrm{H}_{38} \mathrm{O}_{5} 383.3$, found 383.4.

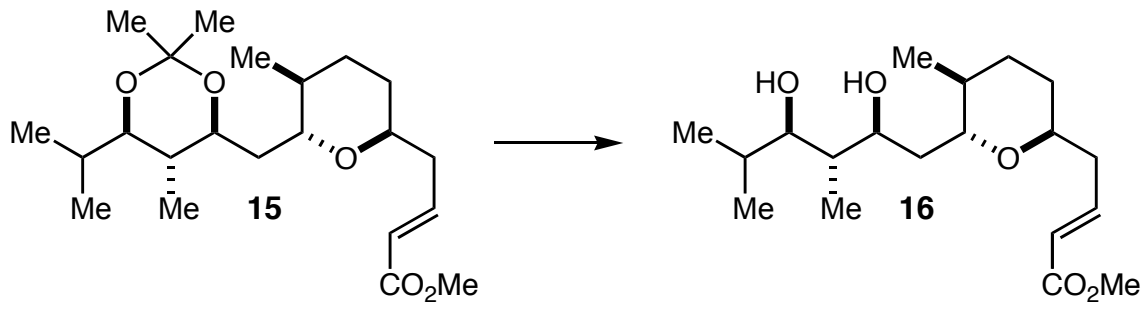

15 (240 mg, $0.628 \mathrm{mmol}$ ) was dissolved in $6.3 \mathrm{~mL}$ of 5:1 AcOH: $\mathrm{H}_{2} \mathrm{O}$, and the resulting solution was heated to $60{ }^{\circ} \mathrm{C}$ for 1 hour. Toluene $(8 \mathrm{~mL})$ was then added, and the mixture was concentrated. The residue was dissolved in EtOAc and washed with saturated aqueous $\mathrm{NaHCO}_{3}$, followed by extraction of the aqueous layer with EtOAc. The combined organic layers were washed with saturated aqueous $\mathrm{NaCl}$, dried over $\mathrm{MgSO}_{4}$, filtered and concentrated to afford 16 (205 mg, 95\% yield). ${ }^{1} \mathrm{H}$ NMR (400 MHz, $\left.\mathrm{CDCl}_{3}\right) \delta 6.94-6.86(\mathrm{~m}, 1 \mathrm{H}$, $\left.\mathrm{CH}=\mathrm{CHCO}_{2} \mathrm{CH}_{3}\right), \quad 5.96-5.89 \quad\left(\mathrm{~m}, \quad 1 \mathrm{H}, \quad \mathrm{CH}=\mathrm{CHCO}_{2} \mathrm{CH}_{3}\right), \quad 4.42 \quad(\mathrm{~s}, \quad 1 \mathrm{H}, \quad \mathrm{OH}), \quad 4.11-4.04 \quad(\mathrm{~m}, \quad 1 \mathrm{H}$, $\left.\mathrm{CH}=\mathrm{CHCH}_{2} \mathrm{CHOR}\right), 3.95(\mathrm{~s}, 1 \mathrm{H}, \mathrm{OH}), 3.74\left(\mathrm{~s}, 3 \mathrm{H}, \mathrm{CO}_{2} \mathrm{CH}_{3}\right), 3.74-3.68(\mathrm{~m}, 1 \mathrm{H}, \mathrm{CHOH}), 3.50-3.42(\mathrm{~m}, 1 \mathrm{H}$, CHOR), $3.34\left(\mathrm{dd}, J_{I}=10.2 \mathrm{~Hz}\right.$ and $\left.J_{2}=2.1 \mathrm{~Hz}, 1 \mathrm{H},\left(\mathrm{CH}_{3}\right)_{2} \mathrm{CHCHOH}\right), 2.75-2.64\left(\mathrm{~m}, 1 \mathrm{H}, \mathrm{CH}_{2} \mathrm{CH}=\mathrm{CHCO}_{2} \mathrm{CH}_{3}\right)$, 2.45-2.36 (m, 1H, $\left.\mathrm{CH}_{2} \mathrm{CH}=\mathrm{CHCO}_{2} \mathrm{CH}_{3}\right), 1.87-1.76\left(\mathrm{~m}, 2 \mathrm{H}, \mathrm{CH}_{2}\right), 1.58-1.30\left(\mathrm{~m}, 6 \mathrm{H}\right.$, five $\mathrm{CH}_{2}$ and $\left.\mathrm{CH}\left(\mathrm{CH}_{3}\right)_{2}\right)$, $1.00\left(\mathrm{~d}, J=6.9 \mathrm{~Hz}, 3 \mathrm{H}, \mathrm{CH}_{3}\right), 0.89\left(\mathrm{~d}, J=6.5 \mathrm{~Hz}, 3 \mathrm{H}, \mathrm{CH}_{3}\right), 0.85\left(\mathrm{~d}, J=6.7 \mathrm{~Hz}, 3 \mathrm{H}, \mathrm{CH}_{3}\right), 0.77(\mathrm{~d}, J=6.7 \mathrm{~Hz}$, $\left.3 \mathrm{H}, \mathrm{CH}_{3}\right) ;{ }^{13} \mathrm{C}$ NMR $\left(100 \mathrm{MHz}, \mathrm{CDCl}_{3}\right) \delta 166.5,144.8,123.5,79.5,78.3,77.7,71.0,51.6,41.5,36.8,35.2$, 34.0, 30.0, 27.3, 26.6, 20.4, 18.1, 14.3, 13.4; IR (thin film) 3435, 2954, 2927, 2874, 2846, 1725, 1658, 1459, 1436, 1379, 1272, 1218, 1164, $1040 \mathrm{~cm}^{-1}$; LRMS (FAB, M+1) calc'd for $\mathrm{C}_{19} \mathrm{H}_{34} \mathrm{O}_{5} 343.2$, found 343.1. 
Page S12

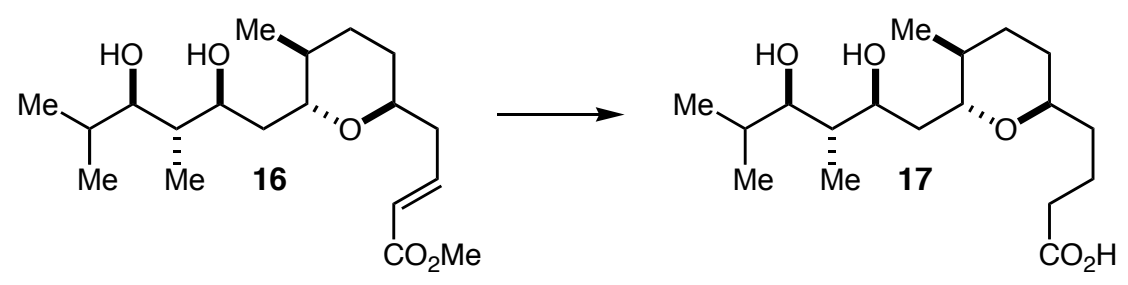

To a solution of $16(240 \mathrm{mg}, 0.628 \mathrm{mmol})$ in $6.0 \mathrm{~mL}$ EtOAc was added $\mathrm{PtO}_{2}(7 \mathrm{mg}, 0.03 \mathrm{mmol})$. An atmosphere of $\mathrm{H}_{2}$ was established (balloon) and the mixture was stirred for 18 hours. The mixture was then filtered through a pad of Celite and and the filtrate was concentrated, affording the corresponding saturated ester (198 mg, 99\% yield). To a solution of the residue $(170 \mathrm{mg}, 0.539 \mathrm{mmol})$ in $3.0 \mathrm{~mL}$ of THF was added $2.5 \mathrm{~mL}$ of $1 \mathrm{~N} \mathrm{NaOH}$. After 15 hours, the $\mathrm{pH}$ of the solution was adjusted to $\sim 2$ by the addition of $1 \mathrm{~N}$ aqueous $\mathrm{NaHSO}_{4}$. The aqueous layer was saturated with $\mathrm{NaCl}$ and extracted three times with EtOAc. The combined organic layers were dried over $\mathrm{MgSO}_{4}$, filtered and concentrated to afford 17 (170 mg, 99\% yield), which was used without further purification. ${ }^{1} \mathrm{H}$ NMR $\left(500 \mathrm{MHz}, \mathrm{CDCl}_{3}\right) \delta$ 3.95-3.86 (m, 2H, two CHOR), 3.52-3.45 (m, 1H, CHOR), $3.36\left(\mathrm{dd}, J_{l}=8.8 \mathrm{~Hz}\right.$ and $\left.J_{2}=2.6 \mathrm{~Hz}, 1 \mathrm{H},\left(\mathrm{CH}_{3}\right)_{2} \mathrm{CHCHOH}\right), 2.44-2.35\left(\mathrm{~m}, 2 \mathrm{H}, \mathrm{CH}_{2} \mathrm{CO}_{2} \mathrm{H}\right), 2.10-1.89(\mathrm{~m}, 2 \mathrm{H}$, $\left.\mathrm{CH}_{2}\right), 1.87-1.72\left(\mathrm{~m}, 3 \mathrm{H}\right.$, three $\left.\mathrm{CH}_{2}\right), 1.69-1.56\left(\mathrm{~m}, 3 \mathrm{H}\right.$, three $\left.\mathrm{CH}_{2}\right), 1.51-1.30\left(\mathrm{~m}, 5 \mathrm{H}\right.$, four $\mathrm{CH}_{2}$ and $\left.\mathrm{CH}\left(\mathrm{CH}_{3}\right)_{2}\right)$, $1.00\left(\mathrm{~d}, J=6.9 \mathrm{~Hz}, 3 \mathrm{H}, \mathrm{CH}_{3}\right), 0.87\left(\mathrm{~d}, J=6.7 \mathrm{~Hz}, 3 \mathrm{H}, \mathrm{CH}_{3}\right), 0.85\left(\mathrm{~d}, J=6.9 \mathrm{~Hz}, 3 \mathrm{H}, \mathrm{CH}_{3}\right), 0.79(\mathrm{~d}, J=6.9 \mathrm{~Hz}$ $\left.3 \mathrm{H}, \mathrm{CH}_{3}\right) ;{ }^{13} \mathrm{C} \mathrm{NMR}\left(100 \mathrm{MHz}, \mathrm{CDCl}_{3}\right) \delta 164.0,79.2,77.7,76.8,72.1,41.6,36.3,35.7,33.5,29.9,28.2,26.9$, 21.1, 20.4, 18.1, 18.1, 14.1, 12.9; IR (thin film) 3443, 2961, 2927, 2873, 2635, 1711, 1453, 1412, 1378, 1242, 1238, $1045 \mathrm{~cm}^{-1}$; LRMS (FAB, M+1) calc'd for $\mathrm{C}_{18} \mathrm{H}_{34} \mathrm{O}_{5} 331.2$, found 331.0.
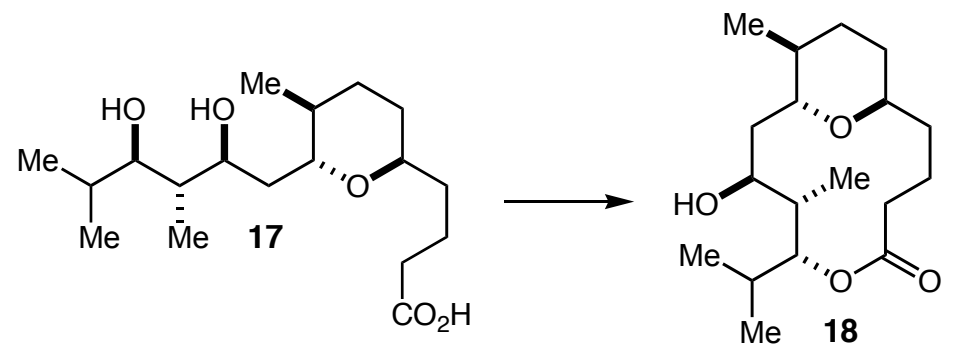

To a solution of $17(85 \mathrm{mg}, 0.247 \mathrm{mmol})$ in $2.0 \mathrm{~mL}$ of toluene was added $\mathrm{Et}_{3} \mathrm{~N}(172 \mu \mathrm{L}, 1.235 \mathrm{mmol})$. After $20 \mathrm{~min}, 2$,4,6-trichlorobenzoyl chloride $(50 \mu \mathrm{L}, 0.321 \mathrm{mmol})$ was then added. After 6 hours the solution was then diluted with $80 \mathrm{~mL}$ of toluene and 4-dimethylaminopyridine (92 mg, $0.75 \mathrm{mmol}$ ) was added. After 1 hour, the reaction mixture was concentrated and the residue was purified by silica gel chromatography $(2 \rightarrow 15 \%$ EtOAc/Hexanes) to afford $18\left(12 \mathrm{mg}, 15 \%\right.$ yield). ${ }^{1} \mathrm{H} \mathrm{NMR}\left(500 \mathrm{MHz}, \mathrm{CDCl}_{3}\right) \delta 4.67\left(\mathrm{dd}, J_{l}=11.0 \mathrm{~Hz}\right.$ and $J_{2}=$ 
$\left.2.1 \mathrm{~Hz}, 1 \mathrm{H},\left(\mathrm{CH}_{3}\right)_{2} \mathrm{CHCHOCOR}\right), 4.15(\mathrm{~s}, 1 \mathrm{H}, \mathrm{OH}), 3.92-3.85(\mathrm{~m}, 1 \mathrm{H}, \mathrm{CHOR}), 3.67\left(\mathrm{dd}, J_{1}=9.9 \mathrm{~Hz}\right.$ and $J_{2}=$ $2.7 \mathrm{~Hz}, 1 \mathrm{H}, \mathrm{CHOH}), 3.26-3.19$ (m, 1H, CHOR), 2.49-2.32 (m, 2H, $\left.\mathrm{CH}_{2} \mathrm{CO}_{2} \mathrm{R}\right), 2.08-2.00(\mathrm{~m}, 1 \mathrm{H}, \mathrm{CH}), 1.95-$ 1.88 (m, 2H, two $\left.\mathrm{CH}_{2}\right), 1.86-1.73$ (m, 2H, two $\left.\mathrm{CH}_{2}\right), 1.78-1.62\left(\mathrm{~m}, 5 \mathrm{H}, \mathrm{CH}_{2}\right), 1.41-1.20$ (m, 4H, $\left.\mathbf{C H}_{2}\right), 0.90(\mathrm{~d}$, $\left.J=6.0 \mathrm{~Hz}, 3 \mathrm{H}, \mathbf{C H}_{3}\right), 0.88\left(\mathrm{~d}, J=6.8 \mathrm{~Hz}, 3 \mathrm{H}, \mathbf{C H}_{3}\right), 0.87\left(\mathrm{~d}, J=7.0 \mathrm{~Hz}, 3 \mathrm{H}, \mathrm{CH}_{3}\right), 0.83(\mathrm{~d}, J=6.8 \mathrm{~Hz}, 3 \mathrm{H}$, $\left.\mathrm{CH}_{3}\right) ;{ }^{13} \mathrm{C} \mathrm{NMR}\left(100 \mathrm{MHz}, \mathrm{CDCl}_{3}\right) \delta 173.3,78.6,78.0,72.5,72.3,39.9,35.9,33.2,32.8,30.1,28.8,27.4,27.0$, 21.5, 20.0, 18.3, 14.4, 9.8; IR (thin film) 3487, 2964, 2933, 2876, 1729, 1462, 1416, 1377, 1249, 1181, 1163, $1083 \mathrm{~cm}^{-1}$; LRMS (FAB, M+1) calc'd for $\mathrm{C}_{18} \mathrm{H}_{32} \mathrm{O}_{4}$ 313.2, found 313.0.

\section{Stereochemical and Regiochemical Proofs:}

1. That the conversion of 6 to 7 resulted in the claimed 14-membered macrolide (as opposed to 12), was easily established by a COSY experiment. The methine proton of the alcohol which underwent esterification is identifiable by its downfield chemical shift, and was found to be coupled to the methine proton of the iso-propyl group.

2. The relative stereochemistry of the THP ring in $\mathbf{9}$ was established by peracetylation of the two alcohols to give 19, which, in combination with a COSY experiment, allowed the assignment of the various methine protons (Scheme 1). Selective 1D NOESY experiments then established the cis 2,6 relative stereochemistry.

\section{Scheme 1}
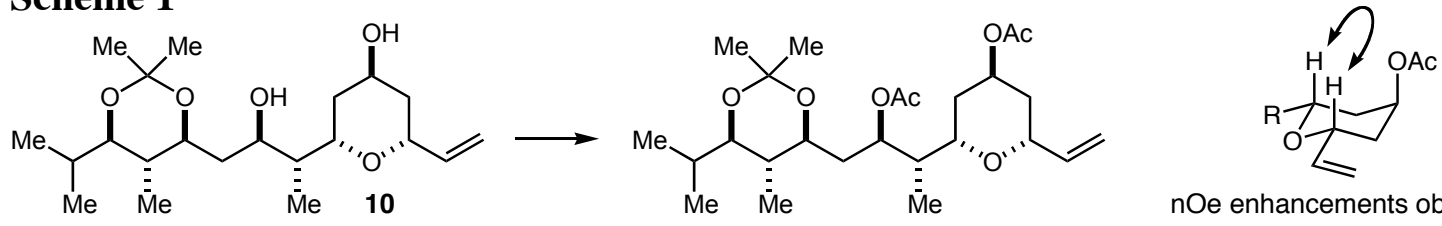

nOe enhancements observed

3. That the conversion of $\mathbf{1 1}$ to $\mathbf{1 2}$ gave the 14-membered macrolide (as opposed to 12), was established by a COSY experiment. The methine proton of the alcohol that underwent esterification is identifiable by its downfield chemical shift, and was found to be coupled to the methine proton of the iso-propyl group.

4. The 2,6-trans relative stereochemistry of the THP ring established in $\mathbf{1 4}$ was established by selective 1D NOESY experiments as described in Scheme 2.

Scheme 2
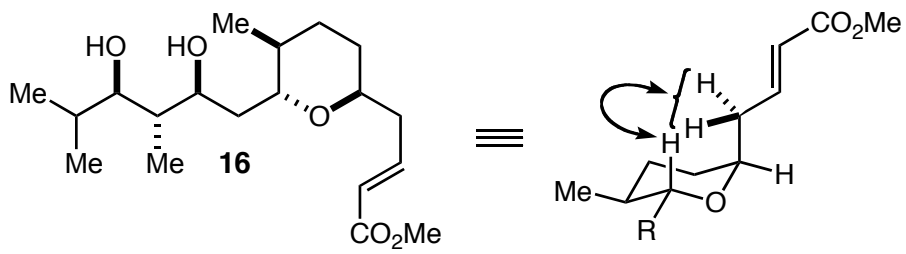


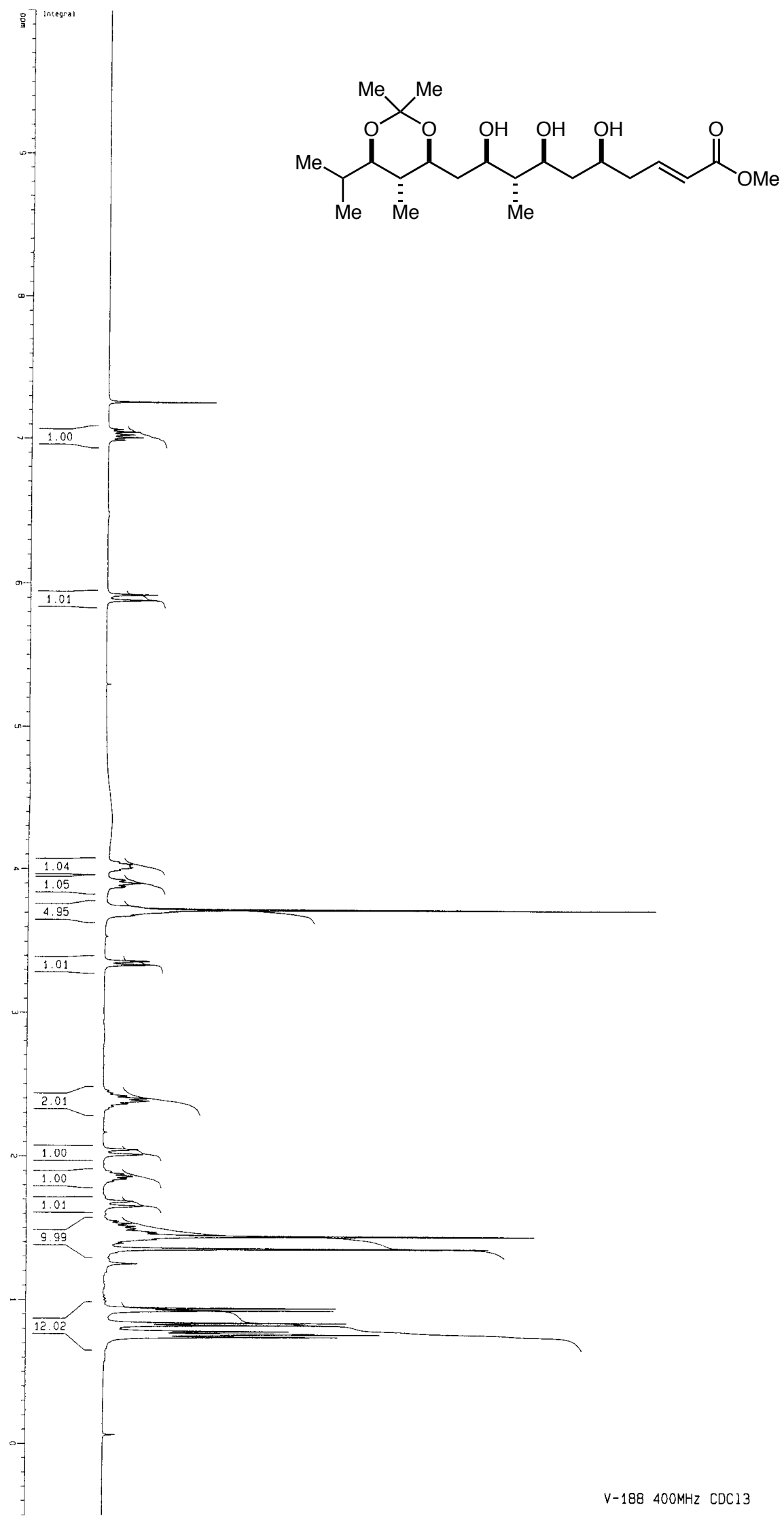

$F^{966.70}$

- 951.96

ل/r 944.35

$\sqrt{-1} \begin{array}{r}818.10 \\ 803.76\end{array}$

If 749.90

If 744.92

UII $r$ - 743.03

$=\sqrt{-374.70}$

$=\Gamma^{560.81}$

519.54

$\int / 519.64$

- 504.68

$-598.50$

-588.73
-585.36

- 576.34

$-567.42$

IL 556.95

II -541.58

$4-499.65$

L 376.71

ᄂ 369.83

- 336.10

-336.27
-312.69

312.61
$-\quad 305.63$

303.28

303.28
-296.60 


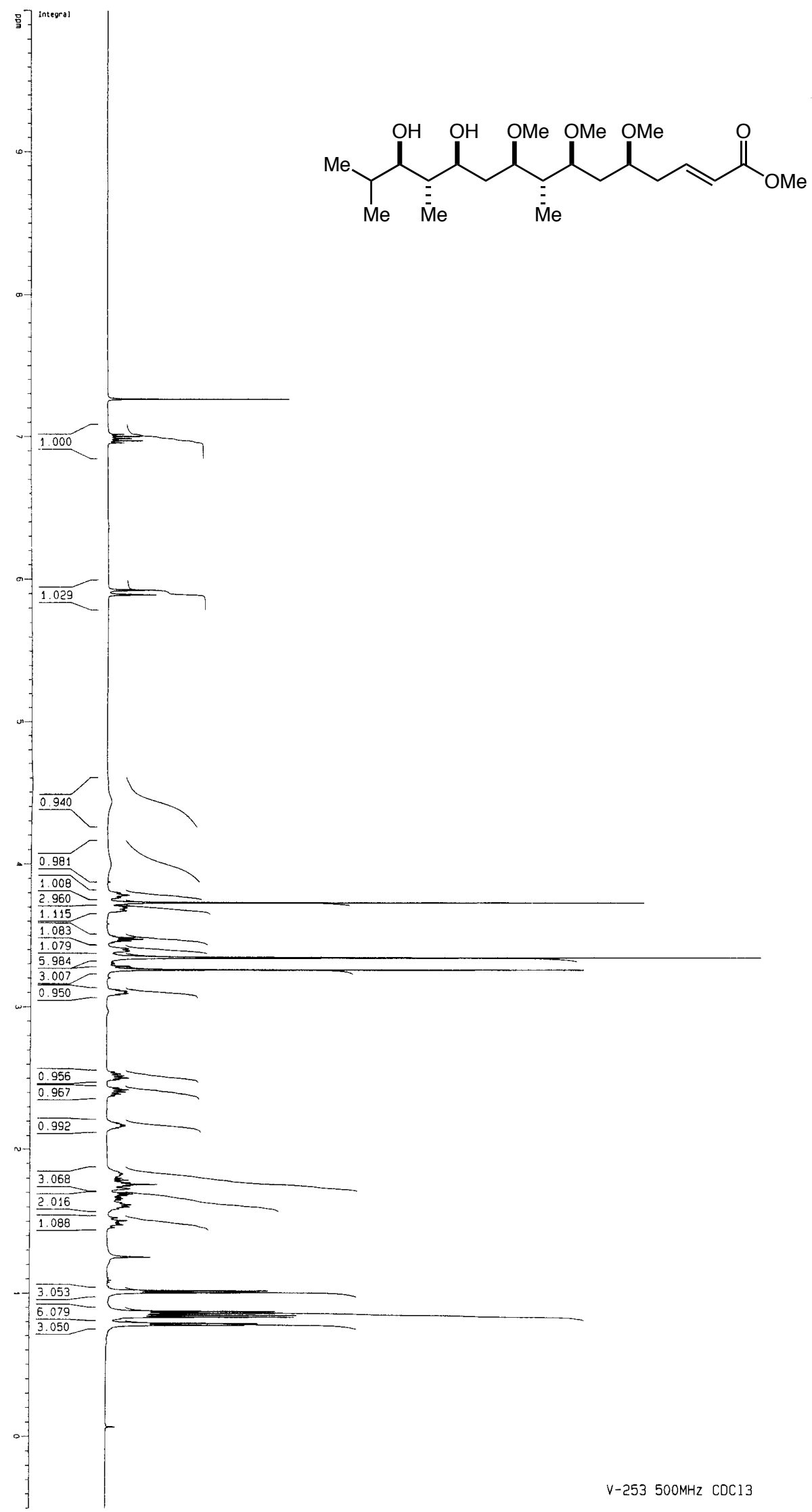

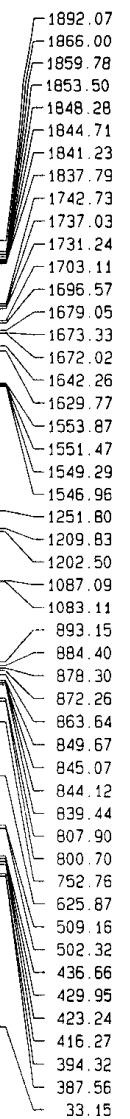



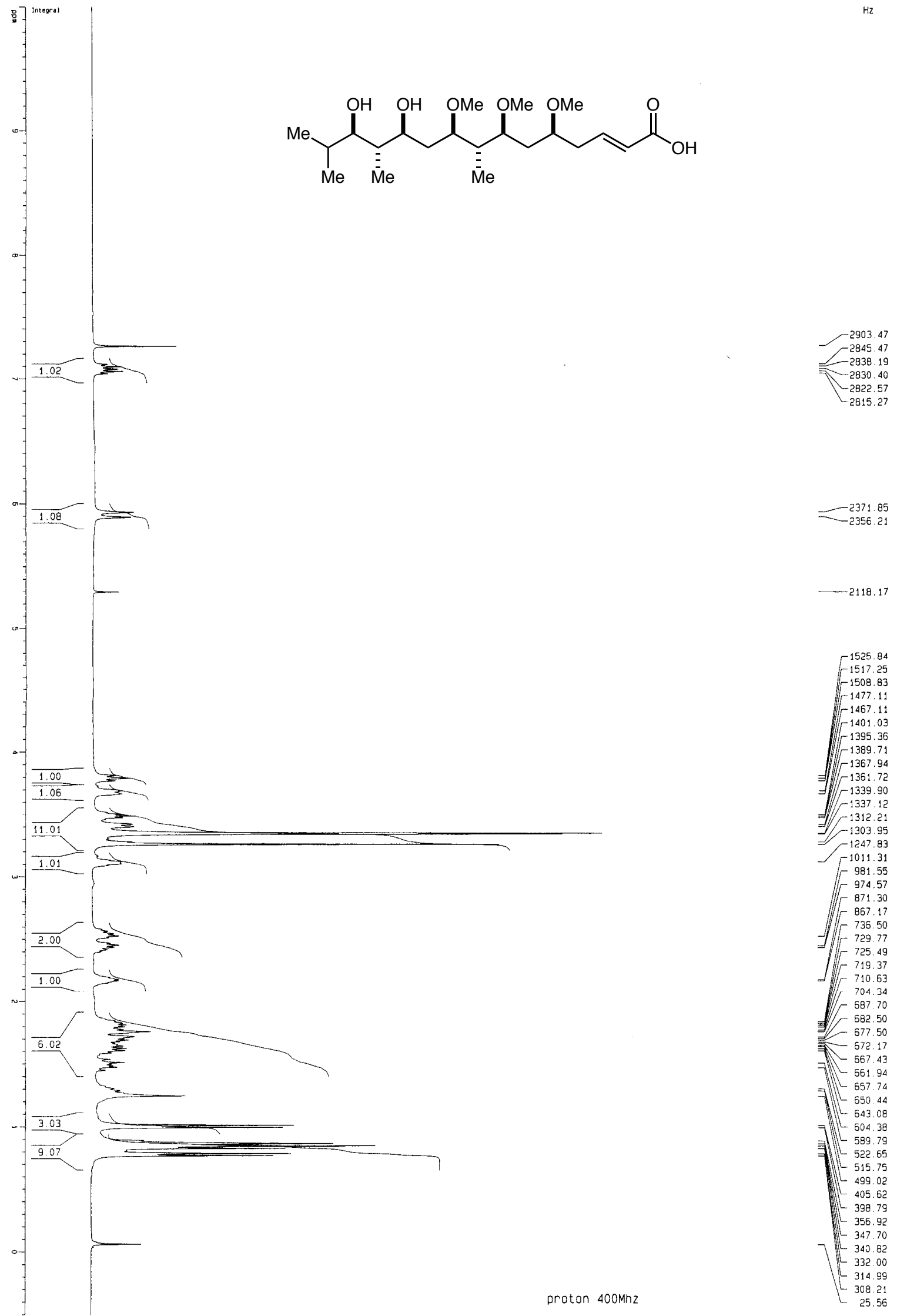

-2371.85
$-\quad 2356.21$

$-2118.17$

1525.84 1517.25
-1508.09 1508.83
-1477.11 $\begin{array}{r}1477.11 \\ 1467.11\end{array}$
-1401.03

$r_{-1401.03}^{1467.11}$

$-1395.36$

-1389.71 $=r^{1367.94}$ $=\int r^{1339.90}$ II $r^{1337.12}$ $=r^{-1312.21}$ $-1247.83$ 101131 1011.31
$-\quad 981.55$ -981.55
$-\quad 974.57$ F 871.30 F 867.17 Jf $\begin{array}{r}736.50 \\ -729.77\end{array}$ ]. 729.77 . $\begin{array}{r}725.49 \\ 719.37\end{array}$ ]. 710.63 IIf 704.34 $\int 1-687.70$ 682.50
-677.50 -572.17
-567.43 - 657.74 7 - 650.44 LL 543,08 7y- 604.38 y- 589.79

522.65
-515.75 L 499.02 $42-405.62$ y- 398.79 FL 356.92 和 347.70 347.70
-340.82 340.82
332.00 332.00
-314.99 308.21
-35.56 proton 400Mnz 
Page S17

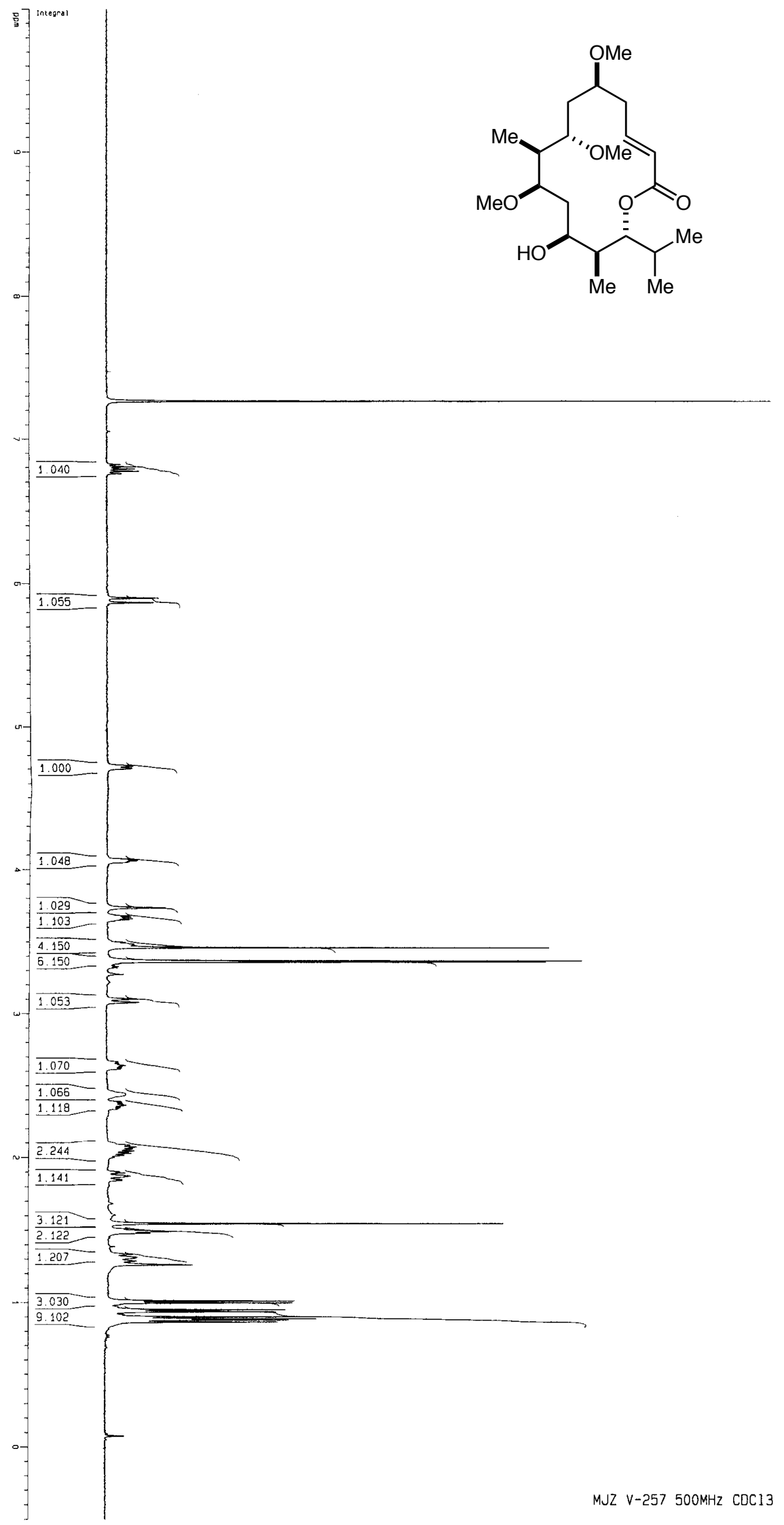

$\mathrm{Hz}$

$-3633.93$

$-3412.16$ 3395.17

$-3388.20$

-2950.82
$-\quad 2934.91$ $r-2354.84$ 2035.72

2031.66
-2026.41

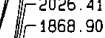

- 1841.33

-1841.33
-1837.20

$-1831.16$

- 1827.21

-1827.21
-1749.12

1749.12
-1742.31

$F_{-1742.31}^{-1738.97}$

fiir -1730.48

ff $r^{1684.90}$

$=\sqrt{-1680.67}$

$=\sqrt{-1551.43}$

$\int \widetilde{\Gamma}^{1551.43}$

$-1320.73$

- 1222.60

1191.01
-1187.08

II $r^{1137.08}$

.II $\Gamma^{-1182.29}$

III -1042.42

- 1035.80

J. $r^{1028.94}$

$r^{1014.01}$

-1007.34

夏 947.85

$-937.13$

925.72

921.59

773.32

754.86
-746.48

$\lfloor 740.30$

-667.39
-657.34

L- 657.34

- 654.37

L 543.46

-505.93
-499.23

-499.23
$-\quad 475.45$

475.4

L 468.19

- 458.19

450.89

444.23
-439.90

$\left[\begin{array}{r}439.90 \\ 432.67\end{array}\right.$ 


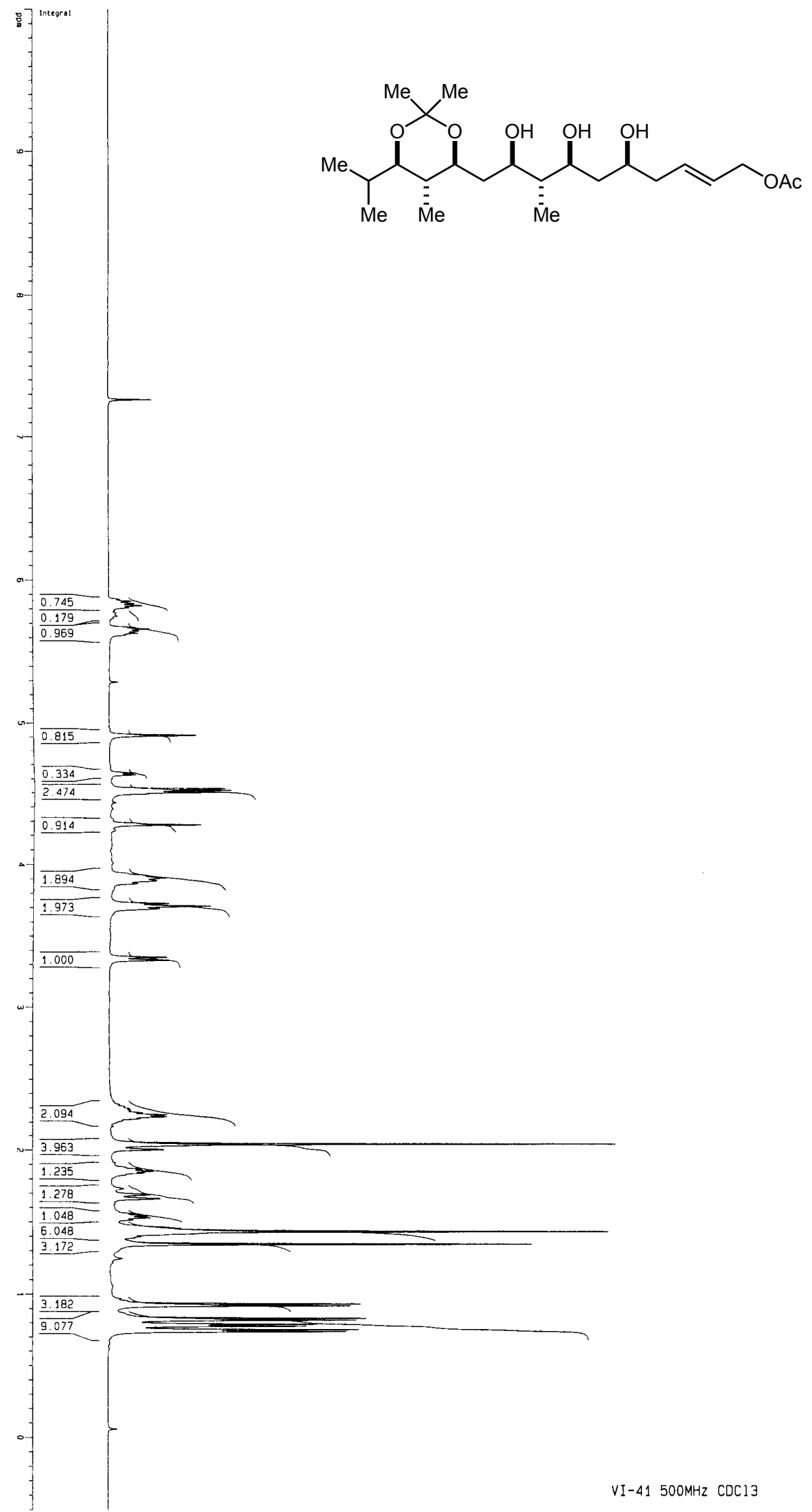


Page S19

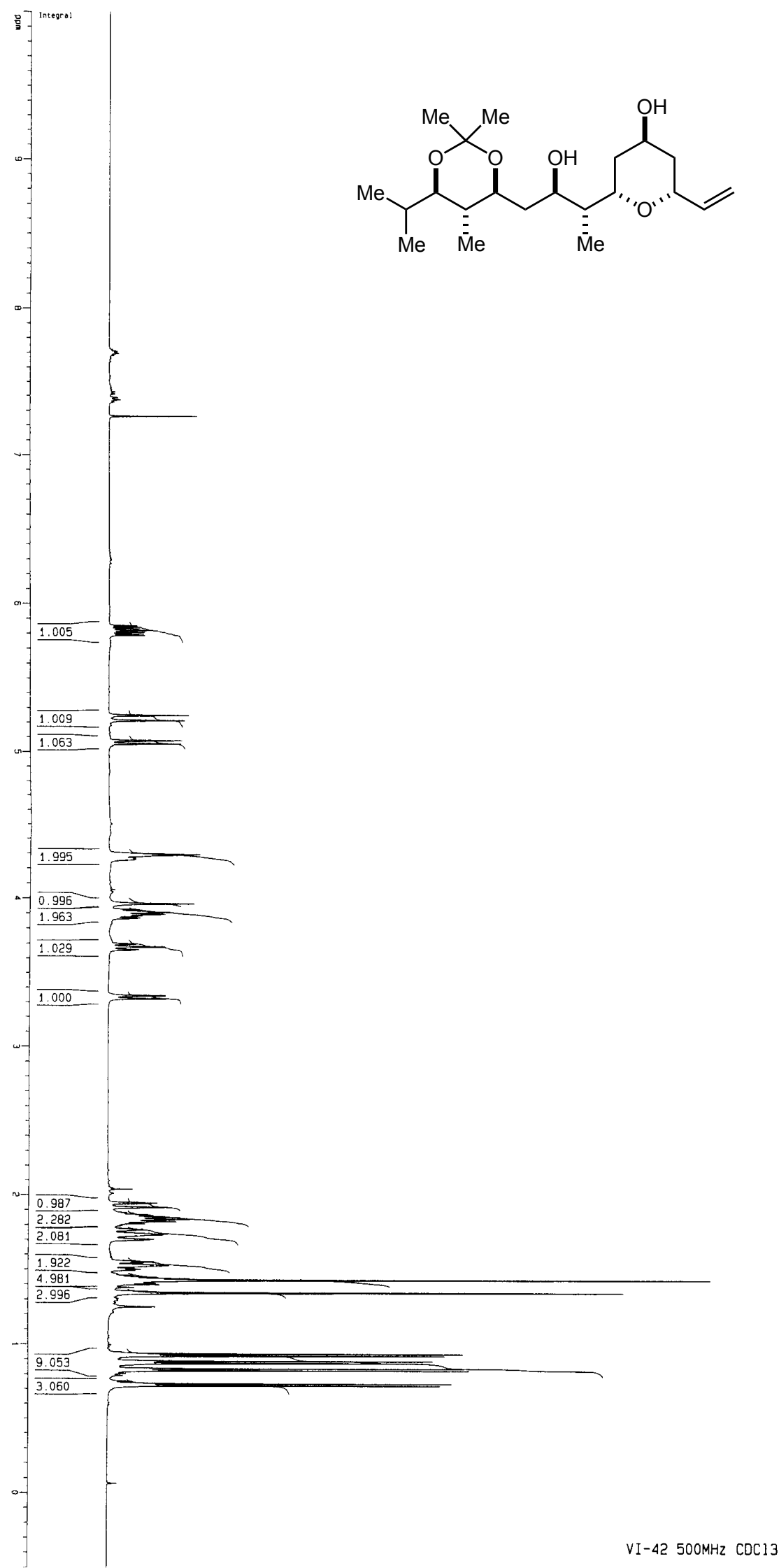

972.37
-958.00

958.00

931.37

926.65

924.42

919.61

917.62

910.87

870.99

f/ 868.15

$=-851.74$

774.49 763.81

$\begin{array}{r}7763.81 \\ \leftarrow \quad 762.25 \\ \hline\end{array}$

ㄴ. 760.20

$-722.54$

718.54

712.95

- 708.59

703.05

L 698.65

$-670.33$

624.28

467.00
460.10

443.02

436.02

436.02
-417.66

410.81

366.40
359.78

$V I-42500 \mathrm{MHz} \quad \mathrm{COC} 13$ 
Page S20

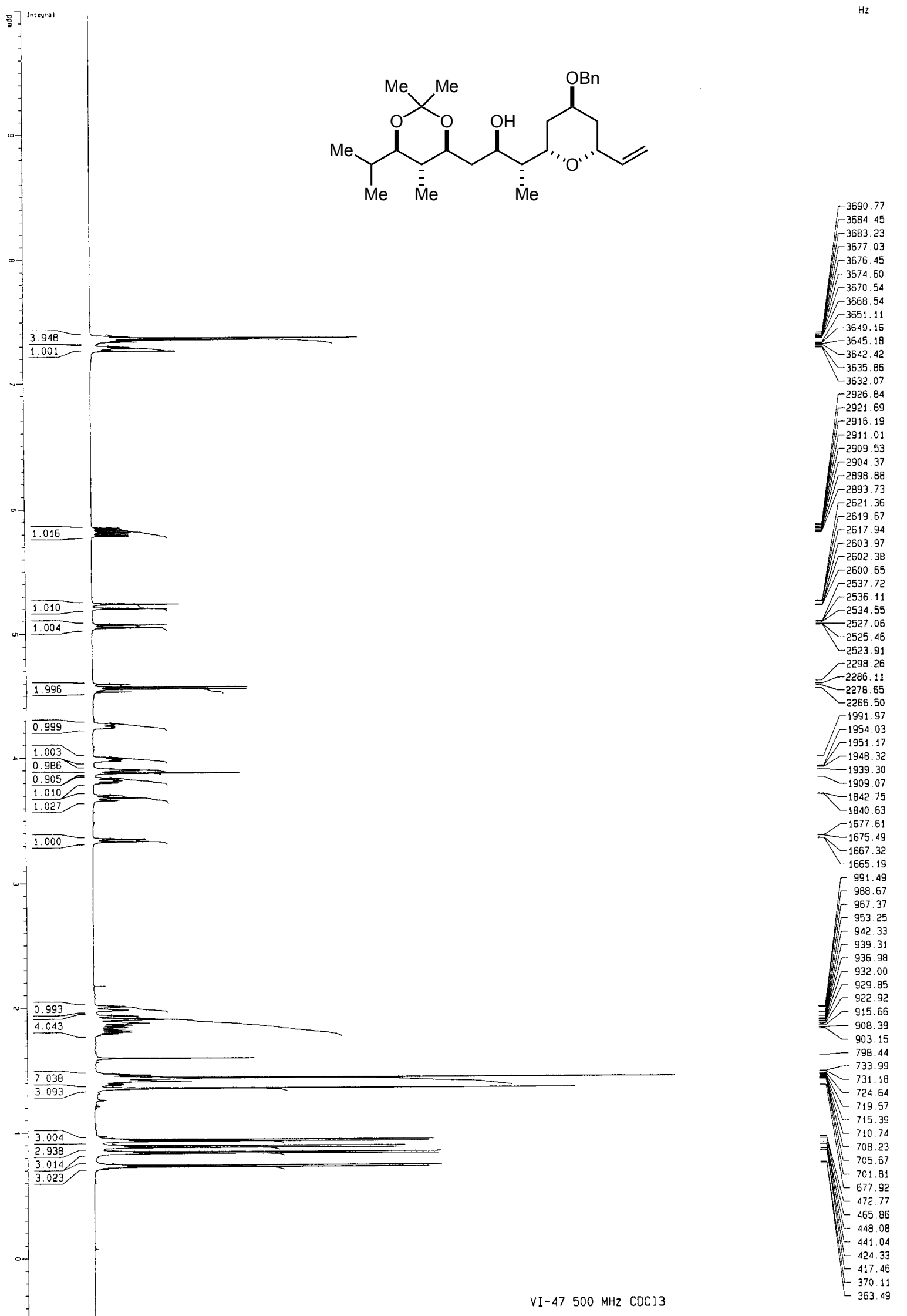


Page S21

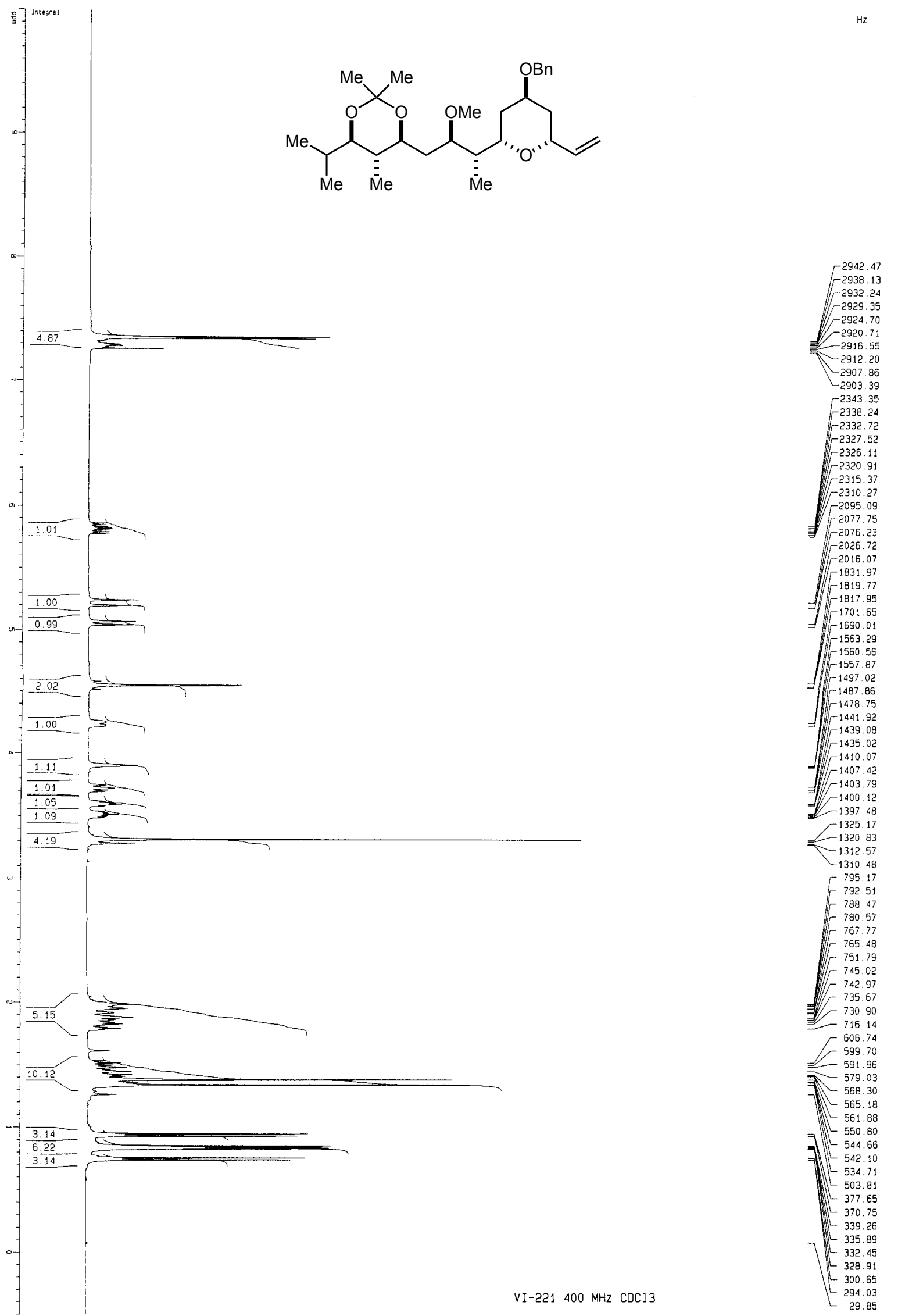


Page S22

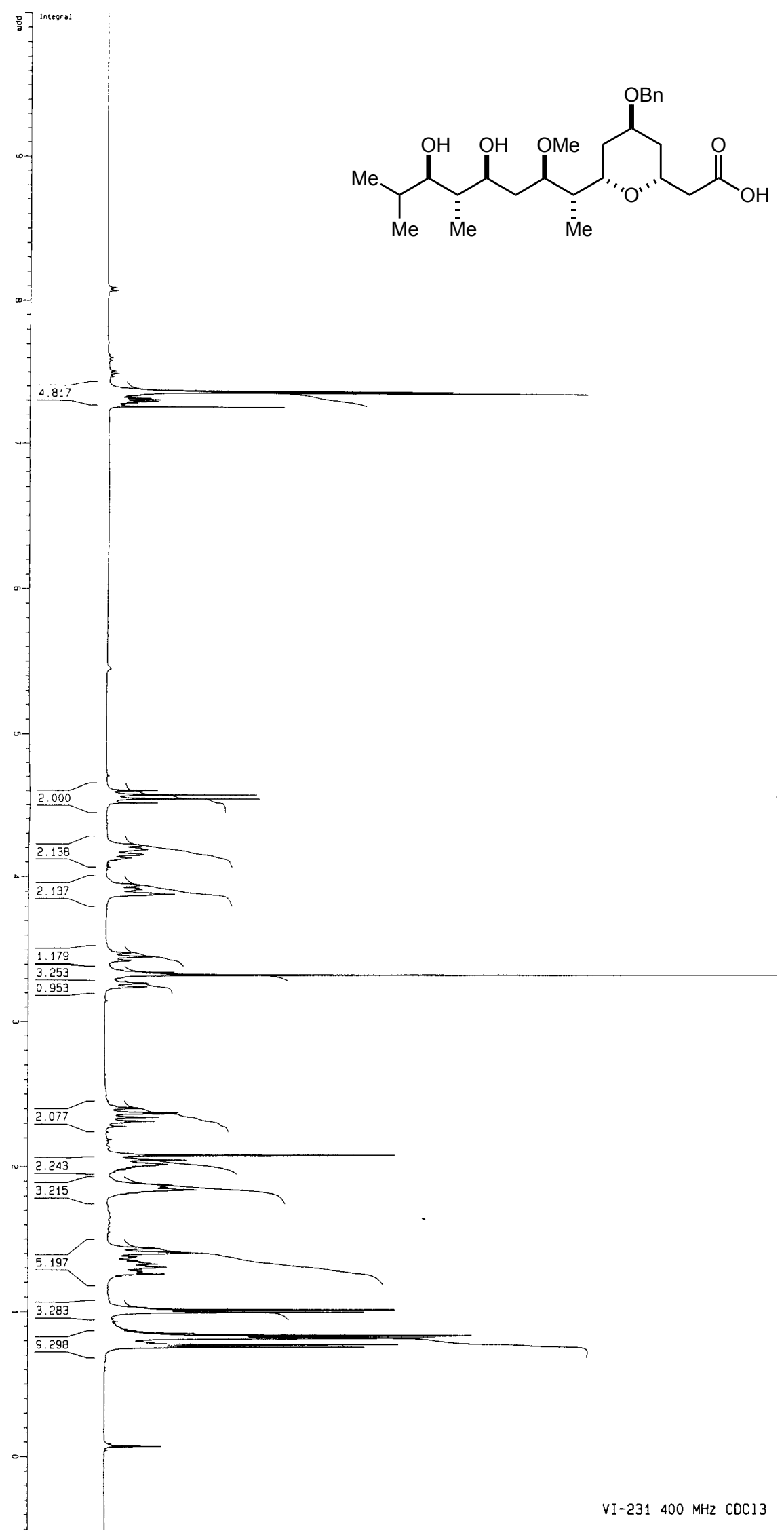

$\Gamma^{1840.69}$ $-1828.46$ $-1817.24$

- 1685.34

If 1674.91

- 1661,82

-1577.34
-1568.92

-1568.09
-1562.09 1562.78
-1555.66 - 1552.96 $\exists \sqrt{\$ 1550.24}$ 1380.68 - ${ }^{1370.68}$ $r_{-1338.28}^{1339}$ $\sqrt{-1329.97}$ - $\Gamma^{1307.98}$ JII ${ }^{1297.36}$ If $\begin{array}{r}963.24 \\ 960.08\end{array}$ If 950.00 949.01
-945.84

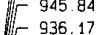

936.17
-925.41

- 830.52

If 817.24

F 805.65 VIf $r \begin{array}{r}749.14 \\ 742.79\end{array}$ $\exists=7472.79$ II $\Gamma_{-736.09}^{729.13}$ $=576.45$ $\int F^{573.88}$ J 562.04 UГ 536.22 IF 533.21 - 530.86 $\begin{array}{r}525.06 \\ -522.21 \\ \hline\end{array}$ 522.21
-516.78 -510.18
-500.23 -508.23
-502.93 $\left[\begin{array}{l}502.93 \\ -419.29\end{array}\right.$ II- 411.29 404.84
-398.00 -398.00
-339.75 - 339.75 334.63
$-\quad 332.92$ 327.83 327.83 325.98
-315.77 315.77 308.40
301.44 301.44
$-\quad 28.12$ 
Page S23

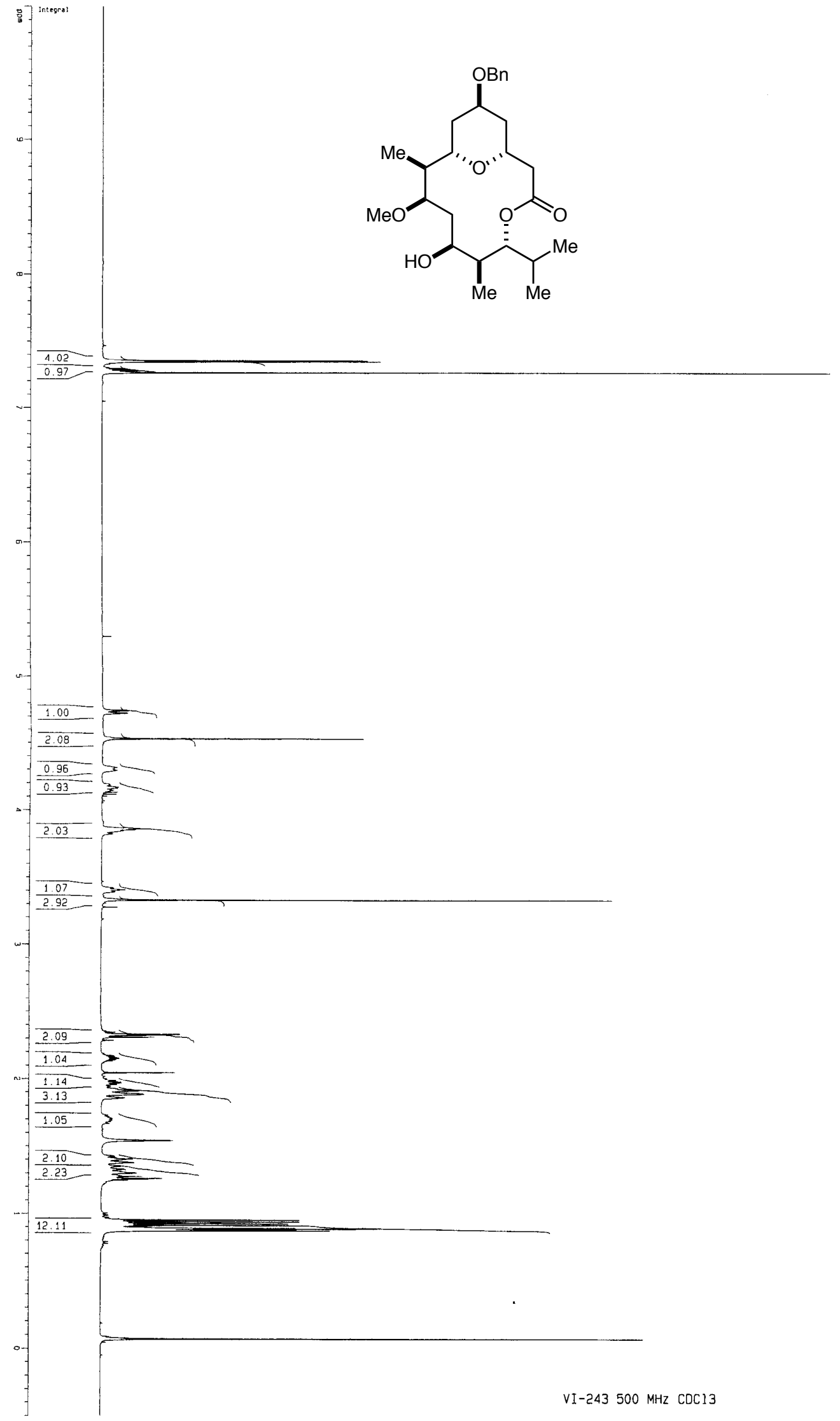


Page S24

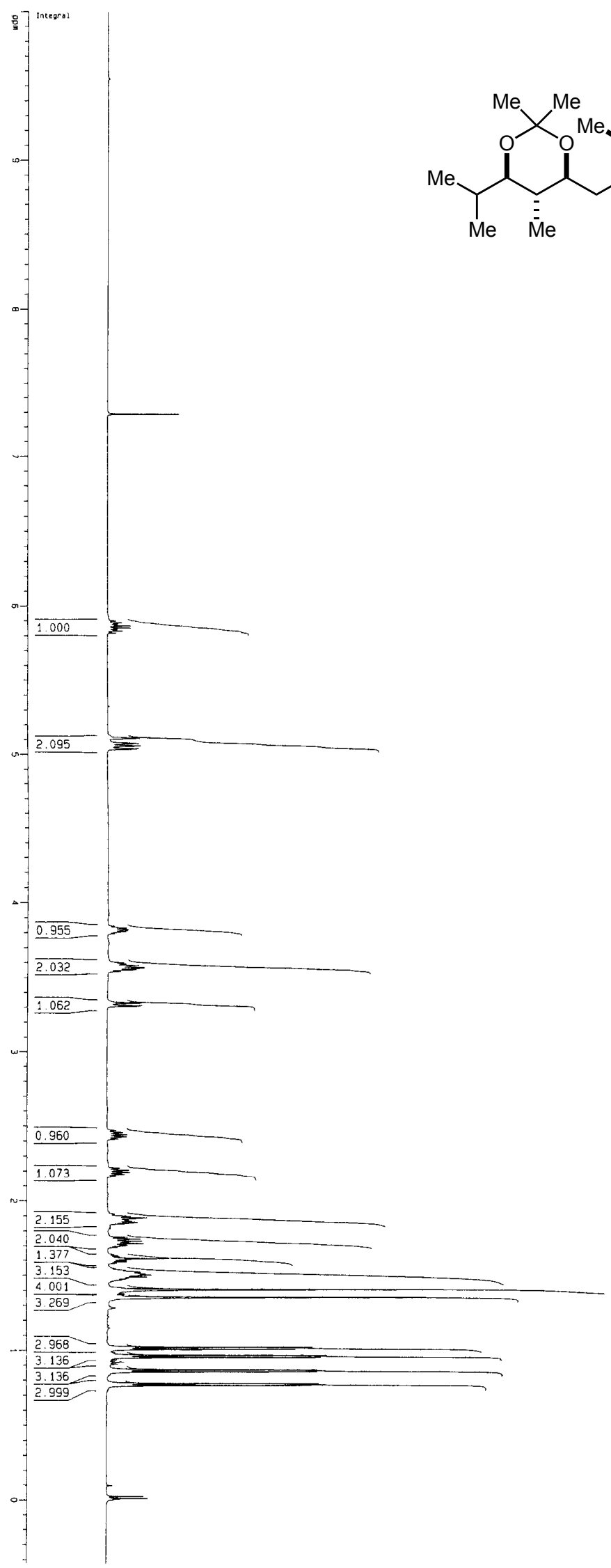

$-3644.24$

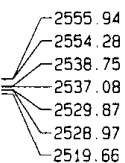

1790.67 $-1783.49$ 1666.21

$-1656.00$

$-1653.93$ F $\begin{array}{r}943.29 \\ 941.59 \\ 932.46\end{array}$ - 932.46

- 928.84

II 872.31

- 865.66

VI ${ }^{857.35}$

$J / r^{805.69}$ $\sqrt{7}{ }^{760} .98$ $\int / \int_{-754.73}^{759.33}$ 752.98 - 75097

I. 74444

L- 701.75

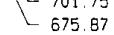

1. 510.03

$=\lfloor-503.23$

$\left[\begin{array}{r}483.21 \\ -476.30\end{array}\right.$

-476.30
-434.70

- 427.85

-388.58
-382.08

_ 11.53

V-268 500MHiz COC13 
Page S25
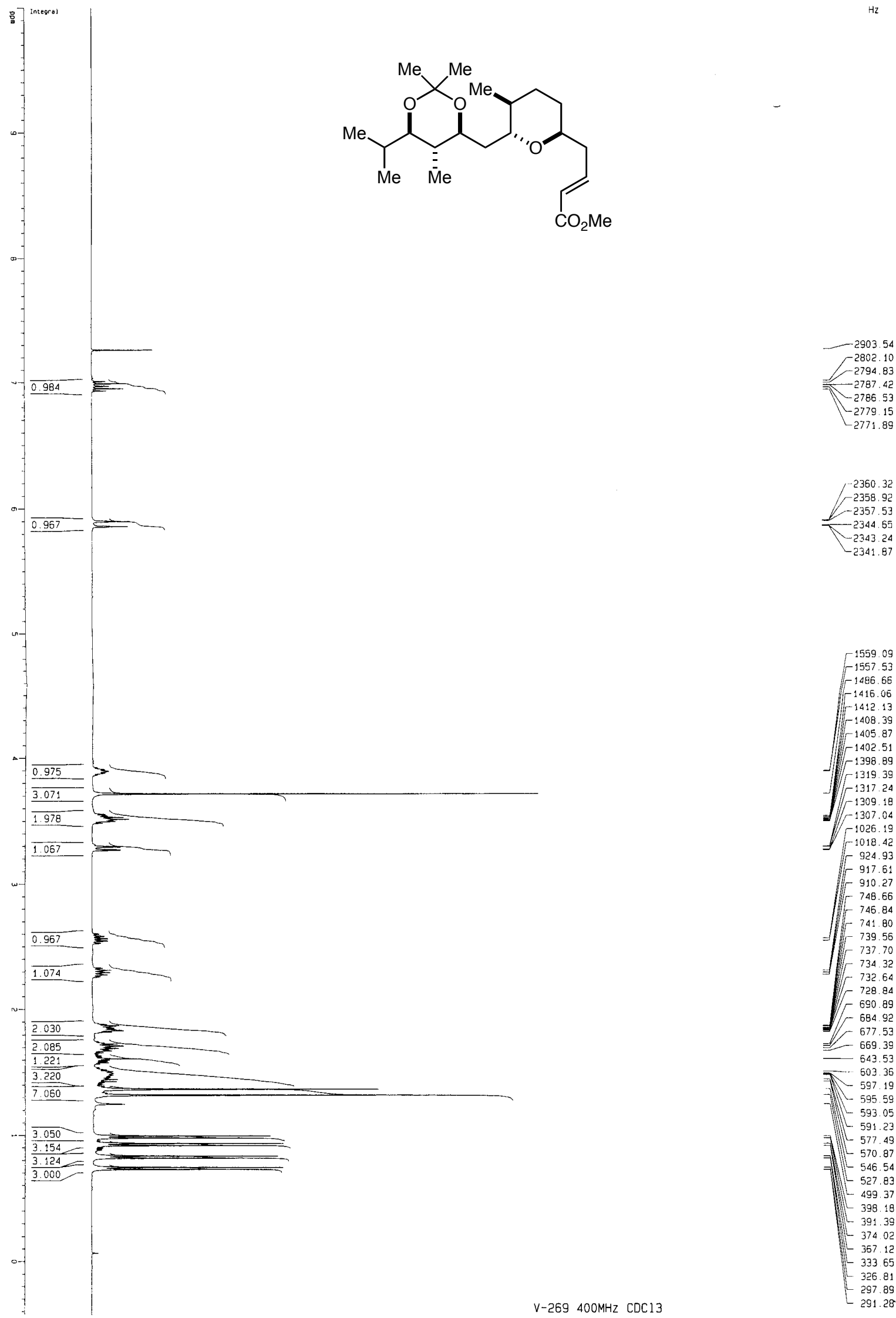

$-1559.09$

-1557.53

1486.66
-1416.05

$r^{1416.06}$

-1412.13
-1408.39

- 1408.39

${ }^{-1402.51}$

-1396.89

$\Gamma^{1319.39}$

$\sqrt{1317.24}$

J $\left.\right|_{-1307.18} ^{1309}$

$-1026.19$

1018.42

- 924.93

917.61
-910.27
-740.60

III -748.66

F 741.80

If $\begin{array}{r}741.80 \\ -739.55\end{array}$

If- 737.70

) 73.32

fir 732.64

- 728.84

I ${ }^{6900.89}$

J// 684.92 $\begin{array}{r}677.53 \\ -669.39 \\ \hline\end{array}$ - 643.53

-... 503.36

$-597.19$

(- 595.59

的 -593.05

$+591.23$

\} $\begin{array}{r}577.49 \\ 570.87\end{array}$

M 570.87

M. 54.54

- 499.37

和 398.18

L. 391.39

L 374.02

- 367.12

- 333.65

- 326.81

$V-269400 \mathrm{MHz} \quad \mathrm{CDC} 13$

297.89 
Page S26

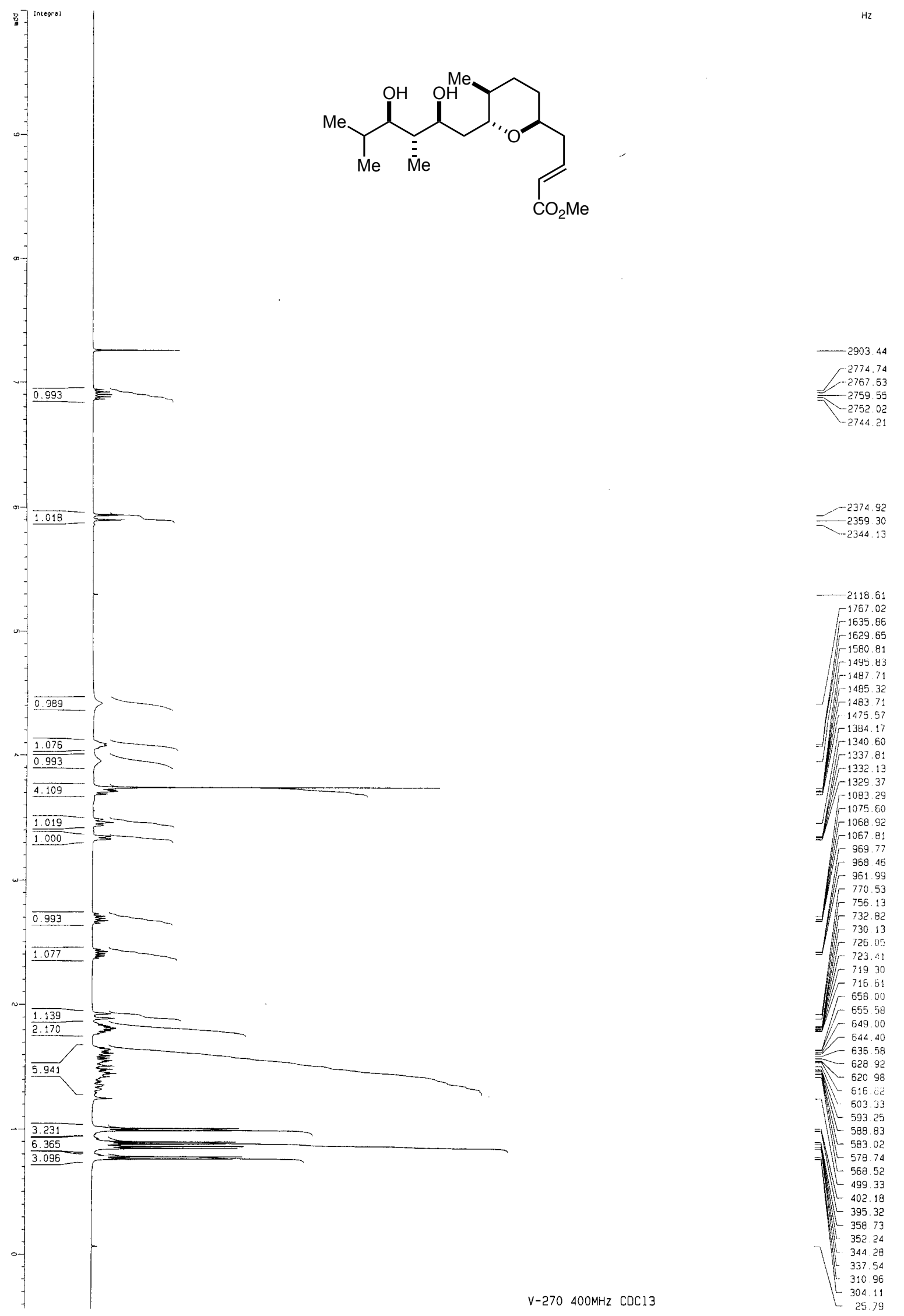


Page S27

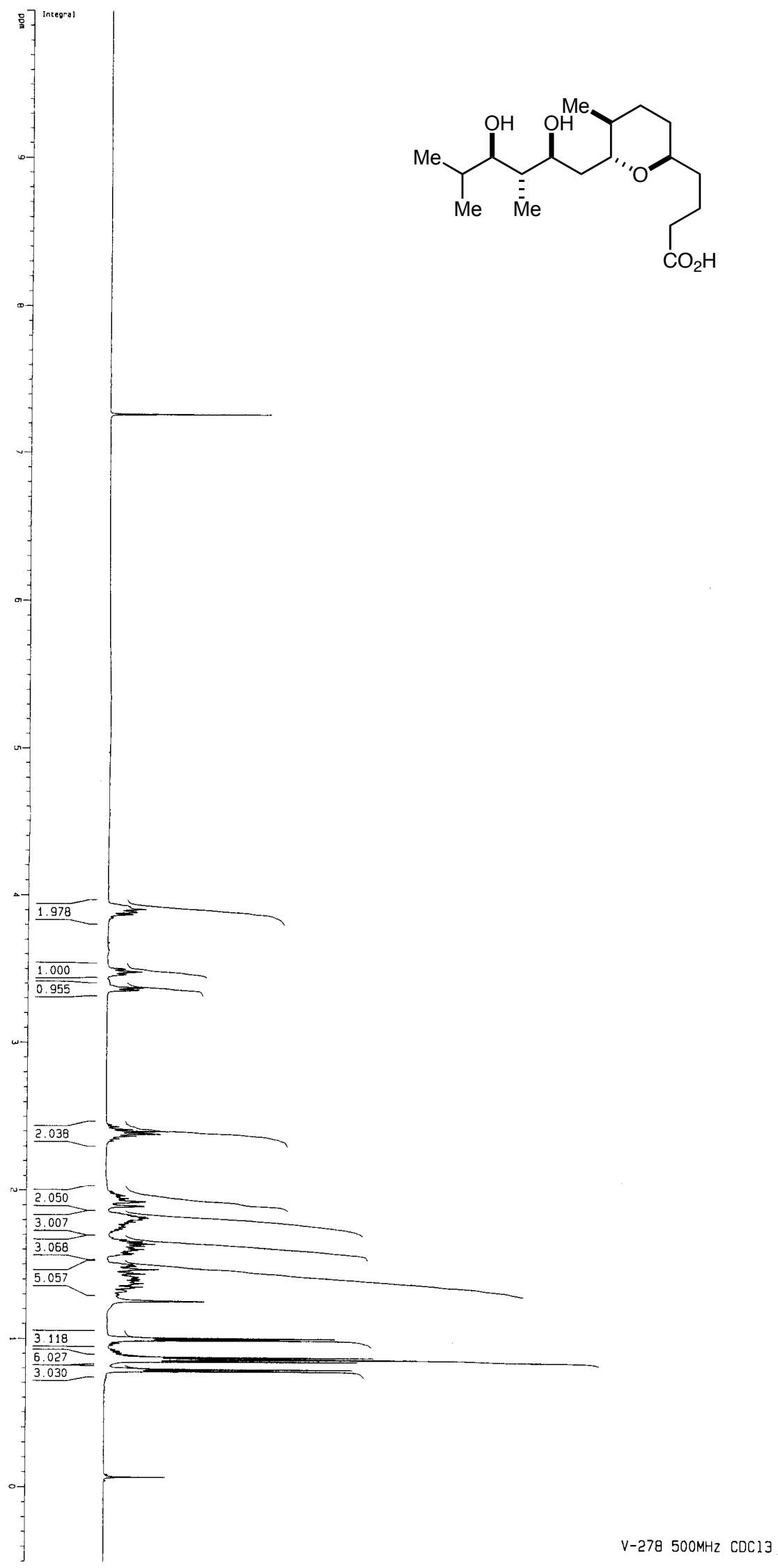


Page S28

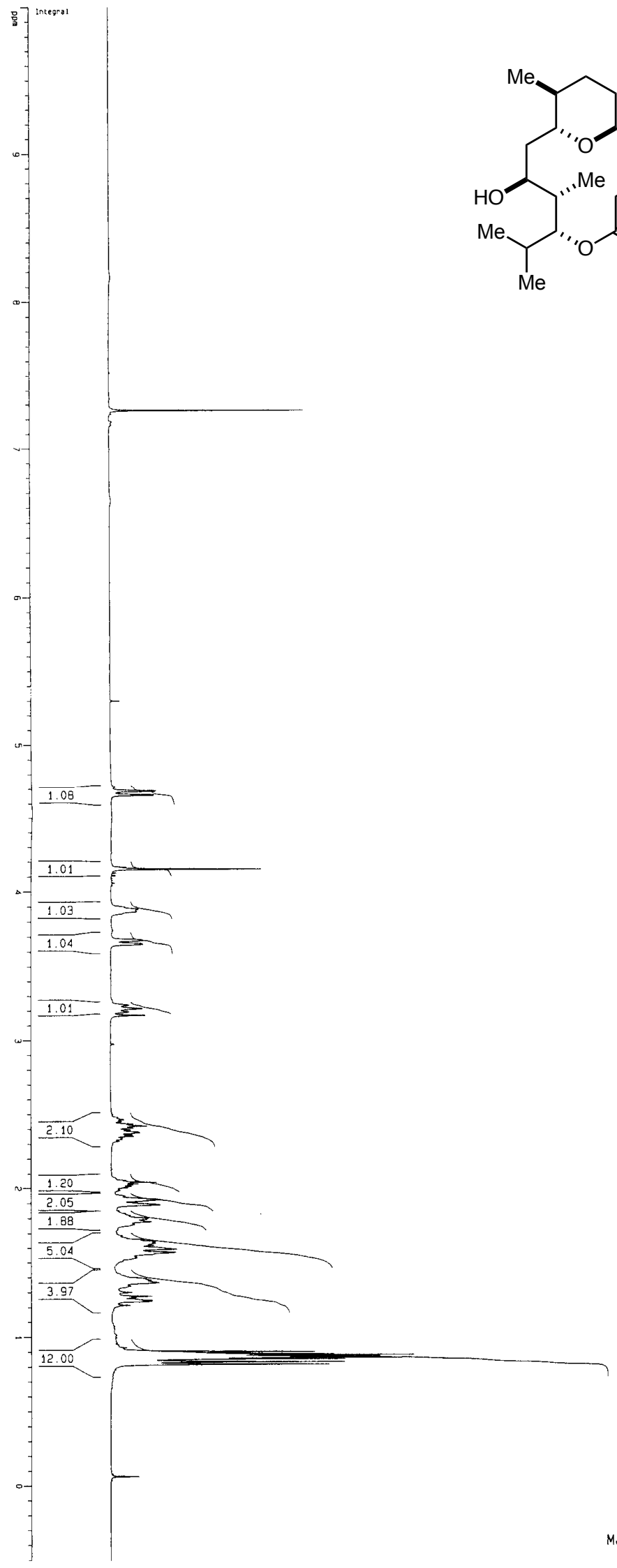

$-2903.4 \mathrm{~B}$

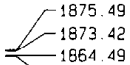

$-1862.40$

$-1660.94$

$-1553.74$ 1472.83
-1470.17 1470.17
-1462.92 $-1460.23$ $-1286.40$ $-1268.02$ T68.83 Tr 953.18

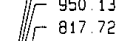
If 811.86

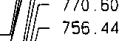
II 720.92 (II) 718.12 JIf $r 711.00$ Jif 663.24 $=1 /$ - 65734 $=\sqrt{653.85}$ - 647.53 $-628.65$ $-546.69$ -546.69
-509.99 -509.99
-502.29 L 499.39 - 495.98 - 361.87

355.88

346.50

342.41

$-334.35$

-327.53
$-\quad 25.72$

MJZ VI-180 400 MHZ $\quad \mathrm{CDCl} 13$ 